\title{
Local Identification of Nonparametric and Semiparametric Models*
}

\author{
Xiaohong Chen \\ Department of Economics \\ Yale \\ Sokbae Lee \\ Department of Economics \\ Seoul National University
}

\author{
Victor Chernozhukov \\ Department of Economics \\ MIT \\ Whitney K. Newey \\ Department of Economics \\ MIT
}

2009

Revised, April 2013

\begin{abstract}
In parametric, nonlinear structural models a classical sufficient condition for local identification, like Fisher (1966) and Rothenberg (1971), is that the vector of moment conditions is differentiable at the true parameter with full rank derivative matrix. We derive an analogous result for the nonparametric, nonlinear structural models, establishing conditions under which an infinite-dimensional analog of the full rank condition is sufficient for local identification. Importantly, we show that additional conditions are often needed in nonlinear, nonparametric models to avoid nonlinearities overwhelming linear effects. We give restrictions on a neighborhood of the true value that are sufficient for local identification. We apply these results to obtain new, primitive identification conditions in several important models, including nonseparable quantile instrumental variable (IV) models, single-index IV models, and semiparametric consumption-based asset pricing models.
\end{abstract}

JEL Classification: C12, C13, C23

Keywords: Identification, Local Identification, Nonparametric Models, Asset Pricing.

${ }^{*}$ The National Science Foundation (Grants SES-0838161 and SES-1132399), European Research Council (ERC-2009-StG-240910-ROMETA), and the National Research Foundation of Korea (NRF-2011-327-B00073) provided financial support for this paper. Helpful comments were provided by a co-editor, three anonymous referees, D. Andrews, D. Chetverikov, K. Evdokimov, J.P. Florens, L. Hansen, J. Heckman, S. Hoderlein, H. Ichimura, T. Komarova, O. Linton, A. Santos and participants in seminars at June 2010 Cowles Conference, June 2010 Beijing Conference, June 2010 LSE Conference, and December 2010 EC2 conference. 


\section{Introduction}

There are many important models in econometrics that give rise to conditional moment restrictions. These restrictions often take the form

$$
\mathrm{E}\left[\rho\left(Y, X, \alpha_{0}\right) \mid W\right]=0,
$$

where $\rho(Y, X, \alpha)$ has a known functional form but $\alpha_{0}$ is unknown. Parametric models (i.e., models when $\alpha_{0}$ is finite dimensional) of this form are well known from the work of Hansen (1982), Chamberlain (1987), and others. Nonparametric versions (i.e., models when $\alpha_{0}$ is infinite dimensional) are motivated by the desire to relax functional form restrictions. Identification and estimation of linear nonparametric conditional moment models have been studied by Newey and Powell (2003), Hall and Horowitz (2005), Blundell, Chen, and Kristensen (2007), Darolles, Fan, Florens, and Renault (2011), and others.

The purpose of this paper is to derive identification conditions for $\alpha_{0}$ when $\rho$ may be nonlinear in $\alpha$ and for other nonlinear nonparametric models. Nonlinear models are important. They include models with conditional quantile restrictions, as discussed in Chernozhukov and Hansen (2005) and Chernozhukov, Imbens, and Newey (2007), and various economic structural and semiparametric models, as further discussed below. In this paper we focus on conditions for local identification of these models. It may be possible to extend these results to provide global identification conditions.

In parametric models there are easily interpretable rank conditions for local identification, as shown in Fisher (1966) and Rothenberg (1971). We give a pair of conditions that are sufficient for parametric local identification from solving a set of equations. They are a) pointwise differentiability at the true value, and b) the rank of the derivative matrix is equal to the dimension of the parameter $\alpha_{0}$. We find that the nonparametric case is different. Differentiability and the nonparametric version of the rank condition may not be sufficient for local identification. We suggest a restriction on the neighborhood that does give local identification, via a link between curvature and an identification set. We also give more primitive conditions for Hilbert spaces, that include interesting econometric examples. In addition we consider semiparametric models, providing conditions for identification of a finite dimensional Euclidean parameter. These 
conditions are based on "partialling out" the nonparametric part and allow for identification of the parametric part even when the nonparametric part is not identified.

The usefulness of these conditions is illustrated by three examples. One example gives primitive conditions for local identification of the nonparametric endogenous quantile models, where primitive identification conditions had only been given previously for discrete regressors. Another example gives conditions for local identification of a semiparametric index model with endogeneity. There we give conditions for identification of parametric components when nonparametric components are not identified. The third example gives sufficient conditions for local identification of a semiparametric consumption capital asset pricing model.

In relation to previous literature, in some cases the nonparametric rank condition is a local version of identification conditions for linear conditional moment restriction models that were considered in Newey and Powell (2003). Chernozhukov, Imbens, and Newey (2007) also suggested differentiability and a rank condition for local identification but did not recognize the need for additional restrictions on the neighborhood. Florens and Sbai (2010) gave local identification conditions for games but their conditions do not apply to the kind of conditional moment restrictions that arise in instrumental variable settings and are a primary subject of this paper.

Section 2 presents general nonparametric local identification results and relates them to sufficient conditions for identification in parametric models. Section 3 gives more primitive conditions for Hilbert spaces and applies them to the nonparametric endogenous quantile model. Section 4 provides conditions for identification in semiparametric models and applies these to the endogenous index model. Section 5 discusses the semiparametric asset pricing example and Section 6 briefly concludes. The Appendix contains additional lemmas and all of the proofs.

\section{Nonparametric Models}

\subsection{The Setting and Definition of Local Identification}

To help explain the nonparametric results and give them context we give a brief description of sufficient conditions for local identification in parametric models. Let $\alpha$ be a $p \times 1$ vector of parameters and $m(\alpha)$ a $J \times 1$ vector of functions with $m\left(\alpha_{0}\right)=0$ for the true value $\alpha_{0}$. Also 
let $|\cdot|$ denote the Euclidean norm in either $\mathbb{R}^{p}$ or $\mathbb{R}^{J}$ depending on the context. We say that $\alpha_{0}$ is locally identified if there is a neighborhood of $\alpha_{0}$ such that $m(\alpha) \neq 0$ for all $\alpha \neq \alpha_{0}$ in the neighborhood. Let $m^{\prime}$ denote the derivative of $m(\alpha)$ at $\alpha_{0}$ when it exists. Sufficient conditions for local identification can be stated as follows:

If $m(\alpha)$ is differentiable at $\alpha_{0}$ and $\operatorname{rank}\left(m^{\prime}\right)=p$ then $\alpha_{0}$ is locally identified.

This statement is proved in the Appendix. Here the sufficient conditions for parametric local identification are pointwise differentiability at the true value $\alpha_{0}$ and the rank of the derivative equal to the number of parameters.

In order to extend these conditions to the nonparametric case we need to modify the notation and introduce structure for infinite dimensional spaces. Let $\alpha$ denote a function with true value $\alpha_{0}$ and $m(\alpha)$ a function of $\alpha$, with $m\left(\alpha_{0}\right)=0$. Conditional moment restrictions are an important example where $\rho(Y, X, \alpha)$ is a finite dimensional residual vector depending on an unknown function $\alpha$ and $m(\alpha)=\mathrm{E}[\rho(Y, X, \alpha) \mid W]$. We impose some mathematical structure by assuming that $\alpha \in \mathcal{A}$, a Banach space with norm $\|\cdot\|_{\mathcal{A}}$ and $m(\alpha) \in \mathcal{B}$, a Banach space with a norm $\|\cdot\|_{\mathcal{B}}$, i.e. $m: \mathcal{A} \mapsto \mathcal{B}$. The restriction of the model is that $\left\|m\left(\alpha_{0}\right)\right\|_{\mathcal{B}}=0$. The notion of local identification we consider is:

Definition: $\alpha_{0}$ is locally identified on $\mathcal{N} \subseteq \mathcal{A}$ if $\|m(\alpha)\|_{\mathcal{B}}>0$ for all $\alpha \in \mathcal{N}$ with $\alpha \neq \alpha_{0}$.

This local identification concept is more general than the one introduced by Chernozhukov, Imbens and Newey (2007). Note that local identification is defined on a set $\mathcal{N}$ in $\mathcal{A}$. Often there exists an $\varepsilon>0$ such that $\mathcal{N}$ is a subset of an open ball

$$
\mathcal{N}_{\varepsilon} \equiv\left\{\alpha \in \mathcal{A}:\left\|\alpha-\alpha_{0}\right\|_{\mathcal{A}}<\varepsilon\right\}
$$

It turns out that it may be necessary for $\mathcal{N}$ to be strictly smaller than an open ball $\mathcal{N}_{\varepsilon}$ in $\mathcal{A}$, as discussed below.

\subsection{Local Identification via Full-Rank Conditions}

The nonparametric version of the derivative will be a bounded (i.e., continuous) linear map $m^{\prime}: \mathcal{A} \mapsto \mathcal{B}$. Under the conditions we give, $m^{\prime}$ will be a Gâteaux derivative at $\alpha_{0}$, that can be 
calculated as

$$
m^{\prime} h=\left.\frac{\partial}{\partial t} m\left(\alpha_{0}+t h\right)\right|_{t=0}
$$

for $h \in \mathcal{A}$ and $t$ a scalar. Sometimes we also require that for any $\delta>0$ there is $\varepsilon>0$ with

$$
\frac{\left\|m(\alpha)-m\left(\alpha_{0}\right)-m^{\prime}\left(\alpha-\alpha_{0}\right)\right\|_{\mathcal{B}}}{\left\|\alpha-\alpha_{0}\right\|_{\mathcal{A}}}<\delta
$$

for all $\alpha \in \mathcal{N}_{\varepsilon}$. This is Fréchet differentiability of $m(\alpha)$ at $\alpha_{0}$ (which implies that the linear map $m^{\prime}: \mathcal{A} \mapsto \mathcal{B}$ is continuous). Fréchet differentiability of estimators that are functionals of the empirical distribution is known to be too strong, but is typically satisfied in local identification analysis, as shown by our examples.

In parametric models the rank condition is equivalent to the null space of the derivative matrix being zero. The analogous nonparametric condition is that the null space of the linear map $m^{\prime}$ is zero, as follows:

Assumption 1 (RAnk Condition): There is a set $\mathcal{N}^{\prime}$ such that $\left\|m^{\prime}\left(\alpha-\alpha_{0}\right)\right\|_{\mathcal{B}}>0$ for all $\alpha \in \mathcal{N}^{\prime}$ with $\alpha \neq \alpha_{0}$.

This condition is familiar from identification of a linear conditional moment model where $Y=\alpha_{0}(X)+U$ and $\mathrm{E}[U \mid W]=0$. Here $\rho(Y, X, \alpha)=Y-\alpha(X)$, so that $m(\alpha)=\mathrm{E}[Y-\alpha(X) \mid W]$ and $m^{\prime} h=-\mathrm{E}[h(X) \mid W]$. In this case Assumption 1 requires that $\mathrm{E}\left[\alpha(X)-\alpha_{0}(X) \mid W\right] \neq 0$ for any $\alpha \in \mathcal{N}^{\prime}$ with $\alpha-\alpha_{0} \neq 0$. For $\mathcal{N}^{\prime}=\mathcal{A}$ this is the completeness condition discussed in Newey and Powell (2003). Andrews (2011) has recently shown that if $X$ and $W$ are continuously distributed, there are at least as many instruments in $W$ as regressors in $X$, and the conditional distribution of $X$ given $W$ is unrestricted (except for a mild regularity condition), then the completeness condition holds generically, in a sense defined in that paper. In Section 3 we also give a genericity result for a different range of models. For this reason we think of Assumption 1 with $\mathcal{N}^{\prime}=\mathcal{A}$ as a weak condition when there are as many continuous instruments $W$ as the endogenous regressors $X$, just as it is in a parametric linear instrumental variables model with unrestricted reduced form. It is also an even weaker condition if some conditions are imposed on the deviations, so in the statement of Assumption 1 we allow it to hold only on $\mathcal{N}^{\prime} \subset \mathcal{A}$. For example, if we restrict $\alpha-\alpha_{0}$ to be a bounded function of $X$, then in linear conditional 
moment restriction models Assumption 1 only requires that the conditional distribution of $X$ given $W$ be bounded complete, which is known to hold for even more distributions than does completeness. This makes Assumption 1 even more plausible in models where $\alpha_{0}$ is restricted to be bounded, such as in Blundell, Chen and Kristensen (2007). See, for example, Mattner (1993), Chernozhukov and Hansen (2005), D'Haultfoeuille (2011), and Andrews (2011) for discussions of completeness and bounded completeness.

Fréchet differentiability and the rank condition are not sufficient for local identification in an open ball $\mathcal{N}_{\varepsilon}$ around $\alpha_{0}$, as we further explain below. One condition that can be added to obtain local identification is that $m^{\prime}: \mathcal{A} \mapsto \mathcal{B}$ is onto.

Theorem 1: If $m(\alpha)$ is Fréchet differentiable at $\alpha_{0}$, the rank condition is satisfied on $\mathcal{N}^{\prime}$ $=\mathcal{N}_{\varepsilon}$ for some $\varepsilon>0$, and $m^{\prime}: \mathcal{A} \mapsto \mathcal{B}$ is onto, then $\alpha_{0}$ is locally identified on $\mathcal{N}_{\tilde{\varepsilon}}$ for some $\tilde{\varepsilon}$ with $0<\tilde{\varepsilon} \leq \varepsilon$.

This result extends previous nonparametric local identification results by only requiring pointwise Fréchet differentiability at $\alpha_{0}$, rather than continuous Fréchet differentiability in a neighborhood of $\alpha_{0}$. This extension may be helpful for showing local identification in nonparametric models, because conditions for pointwise Fréchet differentiability are simpler than for continuous differentiability in nonparametric models.

Unfortunately, the assumption that $m^{\prime}$ is onto is too strong for many econometric models, including many nonparametric conditional moment restrictions. An onto $m^{\prime}$ implies that $m^{\prime}$ has a continuous inverse, by the Banach Inverse Theorem (Luenberger, 1969, p. 149). The inverse of $m^{\prime}$ may not be continuous for nonparametric conditional moment restrictions, as discussed in Newey and Powell (2003). Indeed, the discontinuity of the inverse of $m^{\prime}$ is a now well known ill-posed inverse problem that has received much attention in the econometrics literature, e.g. see the survey of Carrasco, Florens, and Renault (2007). Thus, in many important econometric models Theorem 1 cannot be applied to obtain local identification.

It turns out that $\alpha_{0}$ may not be locally identified on any open ball in ill-posed inverse problems, as we show in an example below. The problem is that for infinite dimensional spaces $m^{\prime}\left(\alpha-\alpha_{0}\right)$ may be small when $\alpha-\alpha_{0}$ is large. Consequently, the effect of nonlinearity, that 
is related to the size of $\alpha-\alpha_{0}$, may overwhelm the identifying effect of nonzero $m^{\prime}\left(\alpha-\alpha_{0}\right)$, resulting in $m(\alpha)$ being zero for $\alpha$ close to $\alpha_{0}$.

We approach this problem by restricting the deviations $\alpha-\alpha_{0}$ to be small when $m^{\prime}\left(\alpha-\alpha_{0}\right)$ is small. The restrictions on the deviations will be related to the nonlinearity of $m(\alpha)$ via the following condition:

Assumption 2: There are $L \geq 0, r \geq 1$ and a set $\mathcal{N}^{\prime \prime}$ such that for all $\alpha \in \mathcal{N}^{\prime \prime}$,

$$
\left\|m(\alpha)-m\left(\alpha_{0}\right)-m^{\prime}\left(\alpha-\alpha_{0}\right)\right\|_{\mathcal{B}} \leq L\left\|\alpha-\alpha_{0}\right\|_{\mathcal{A}}^{r}
$$

This condition is general. It includes the linear case where $\mathcal{N}^{\prime \prime}=\mathcal{A}$ and $L=0$. It also includes Fréchet differentiability, where $r=1, L$ is any positive number and $\mathcal{N}^{\prime \prime}=\mathcal{N}_{\varepsilon}$ for any sufficiently small $\varepsilon>0$. Cases with $r>1$, are analogous to Hölder continuity of the derivative in finite dimensional spaces, with $r=2$ corresponding to twice continuous Fréchet differentiability. We would only have $r>2$ when the second derivative is zero. This condition applies to many interesting examples, as we will show in the rest of the paper. The term $L\left\|\alpha-\alpha_{0}\right\|_{\mathcal{A}}^{r}$ represents a magnitude of nonlinearity that is allowed for $\alpha \in \mathcal{N}^{\prime \prime}$. The following result uses Assumption 2 to specify restrictions on $\alpha$ that are sufficient for local identification.

Theorem 2: If Assumption 2 is satisfied then $\alpha_{0}$ is locally identified on $\mathcal{N}=\mathcal{N}^{\prime \prime} \cap \mathcal{N}^{\prime \prime \prime}$ with $\mathcal{N}^{\prime \prime \prime}=\left\{\alpha:\left\|m^{\prime}\left(\alpha-\alpha_{0}\right)\right\|_{\mathcal{B}}>L\left\|\alpha-\alpha_{0}\right\|_{\mathcal{A}}^{r}\right\}$.

The strict inequality in $\mathcal{N}^{\prime \prime \prime}$ is important for the result. It does exclude $\alpha_{0}$ from $\mathcal{N}$, but that works because local identification specifies what happens when $\alpha \neq \alpha_{0}$. This result includes the linear case, where $L=0, \mathcal{N}^{\prime \prime}=\mathcal{A}$, and $\mathcal{N}=\mathcal{N}^{\prime \prime \prime}=\mathcal{N}^{\prime}$. It also includes nonlinear cases where only Fréchet differentiability is imposed, with $r=1$ and $L$ equal to any positive constant. In that case $\mathcal{N}^{\prime \prime}=\mathcal{N}_{\varepsilon}$ for some $\varepsilon$ small enough and $\alpha \in \mathcal{N}^{\prime \prime \prime}$ restricts $\alpha-\alpha_{0}$ to a set where the inverse of $m^{\prime}$ is continuous by requiring that $\left\|m^{\prime}\left(\alpha-\alpha_{0}\right)\right\|_{\mathcal{B}}>L\left\|\alpha-\alpha_{0}\right\|_{\mathcal{A}}$. In general, by $L\left\|\alpha-\alpha_{0}\right\|_{\mathcal{A}}^{r} \geq 0$, we have $\mathcal{N}^{\prime \prime \prime} \subseteq \mathcal{N}^{\prime}$ for $\mathcal{N}^{\prime}$ from Assumption 1, so the rank condition is imposed by restricting attention to the $\mathcal{N}$ of Theorem 2. Here the rank condition is still important, since if it is not satisfied on some interesting set $\mathcal{N}^{\prime}$, Theorem 2 cannot give local identification on an interesting set $\mathcal{N}$. 
Theorem 2 forges a link between the curvature of $m(\alpha)$ as in Assumption 2 and the identification set $\mathcal{N}$. An example is a scalar $\alpha$ and twice continuously differentiable $m(\alpha)$ with bounded second derivative. Here Assumption 2 will be satisfied with $r=2, L=\sup _{\alpha}\left|d^{2} m(\alpha) / d \alpha^{2}\right| / 2$, and $\mathcal{N}^{\prime \prime}$ equal to the real line, where $|\cdot|$ denotes the absolute value. Assumption 1 will be satisfied with $\mathcal{N}^{\prime}$ equal to the real line as long as $m^{\prime}=d m\left(\alpha_{0}\right) / d \alpha$ is nonzero. Then $\mathcal{N}^{\prime \prime \prime}=\left\{\alpha:\left|\alpha-\alpha_{0}\right|<L^{-1}\left|m^{\prime}\right|\right\}$. Here $L^{-1}\left|m^{\prime}\right|$ is the minimum distance $\alpha$ must go from $\alpha_{0}$ before $m(\alpha)$ can "bend back" to zero. In nonparametric models $\mathcal{N}^{\prime \prime \prime}$ will be an analogous set.

When $r=1$ the set $\mathcal{N}^{\prime \prime \prime}$ will be a linear cone with vertex at $\alpha_{0}$, which means that if $\alpha \in \mathcal{N}^{\prime \prime \prime}$ then so is $\lambda \alpha+(1-\lambda) \alpha_{0}$ for $\lambda>0$. In general, $\mathcal{N}^{\prime \prime \prime}$ is not convex, so it is not a convex cone. For $r>1$ the set $\mathcal{N}^{\prime \prime \prime}$ is not a cone although it is star shaped around $\alpha_{0}$, meaning that for any $\alpha \in \mathcal{N}^{\prime \prime \prime}$ we have $\lambda \alpha+(1-\lambda) \alpha_{0} \in \mathcal{N}^{\prime \prime \prime}$ for $0<\lambda \leq 1$.

Also, if $r>1$ then for any $L>0$ and $1 \leq r^{\prime}<r$ there is $\delta>0$ such that

$$
\mathcal{N}_{\delta} \cap\left\{\alpha:\left\|m^{\prime}\left(\alpha-\alpha_{0}\right)\right\|_{\mathcal{B}}>L\left\|\alpha-\alpha_{0}\right\|_{\mathcal{A}}^{r^{\prime}}\right\} \subseteq \mathcal{N}_{\delta} \cap \mathcal{N}^{\prime \prime \prime}
$$

In this sense $\alpha \in \mathcal{N}^{\prime \prime \prime}$ as assumed in Theorem 2 is less restrictive the larger is $r$, i.e. the local identification neighborhoods of Theorem 2 are "richer" the larger is $r$.

\subsection{Discussion of Assumptions 1 and 2}

Restricting the set of $\alpha$ to be smaller than an open ball can be necessary for local identification in nonparametric models, as we now show in an example. Suppose $\alpha=\left(\alpha_{1}, \alpha_{2}, \ldots\right)$ is a sequence of real numbers. Let $\left(p_{1}, p_{2}, \ldots\right)$ be probabilities, $p_{j}>0, \sum_{j=1}^{\infty} p_{j}=1$. Let $f(x)$ be a twice continuously differentiable function of a scalar $x$ that is bounded with bounded second derivative. Suppose $f(x)=0$ if and only if $x \in\{0,1\}$ and $d f(0) / d x=1$. Let $m(\alpha)=\left(f\left(\alpha_{1}\right), f\left(\alpha_{2}\right), \ldots\right)$

also be a sequence with $\|m(\alpha)\|_{\mathcal{B}}=\left(\sum_{j=1}^{\infty} p_{j} f\left(\alpha_{j}\right)^{2}\right)^{1 / 2}$. Then for $\|\alpha\|_{\mathcal{A}}=\left(\sum_{j=1}^{\infty} p_{j} \alpha_{j}^{4}\right)^{1 / 4}$ the function $m(\alpha)$ will be Fréchet differentiable at $\alpha_{0}=0$, with $m^{\prime} h=h$. A fourth moment norm for $\alpha$, rather than a second moment norm, is needed to make $m(\alpha)$ Fréchet differentiable under the second moment norm for $m(\alpha)$. Here the map $m^{\prime}$ is not onto, even though it is the identity, because the norm on $\mathcal{A}$ is stronger than the norm on $\mathcal{B}$.

In this example the value $\alpha_{0}=0$ is not locally identified by the equation $m(\alpha)=0$ on any open ball in the norm $\left\|\alpha-\alpha_{0}\right\|_{\mathcal{A}}$. To show this result consider $\alpha^{k}$ which has zeros in the 
first $k$ positions and a one everywhere else, i.e., $\alpha^{k}=(0, \ldots, 0,1,1, \ldots)$. Then $m\left(\alpha^{k}\right)=0$ and for $\Delta^{k}=\sum_{j=k+1}^{\infty} p_{j} \longrightarrow 0$ we have $\left\|\alpha^{k}-\alpha_{0}\right\|_{\mathcal{A}}=\left(\sum_{j=1}^{\infty} p_{j}\left[\alpha_{j}^{k}\right]^{4}\right)^{1 / 4}=\left(\Delta^{k}\right)^{1 / 4} \longrightarrow 0$. Thus, we have constructed a sequence of $\alpha^{k}$ not equal to $\alpha_{0}$ such that $m\left(\alpha^{k}\right)=0$ and $\left\|\alpha^{k}-\alpha_{0}\right\|_{\mathcal{A}} \longrightarrow 0$.

We can easily describe the set $\mathcal{N}$ of Theorem 2 in this example, on which $\alpha_{0}=0$ will be locally identified. By the second derivative of $f$ being bounded, Assumption 2 is satisfied with $\mathcal{N}^{\prime \prime}=\mathcal{A}, r=2$, and $L=\sup _{a}\left|\partial^{2} f(a) / \partial a^{2}\right| / 2$, where $L \geq 1$ by the fact that $f^{\prime}(0)=1$ and $f(0)=f(1)=0\left(\right.$ an expansion gives $0=f(1)=1+2^{-1} \partial^{2} f(\bar{a}) / \partial a^{2}$ for $\left.0 \leq \bar{a} \leq 1\right)$. Then,

$$
\mathcal{N}=\left\{\alpha=\left(\alpha_{1}, \alpha_{2}, \ldots\right):\left(\sum_{j=1}^{\infty} p_{j} \alpha_{j}^{2}\right)^{1 / 2}>L\left(\sum_{j=1}^{\infty} p_{j} \alpha_{j}^{4}\right)^{1 / 2}\right\} .
$$

The sequence $\left(\alpha^{k}\right)_{k=1}^{\infty}$ given above will not be included in this set because $L \geq 1$. A simple subset of $\mathcal{N}$ (on which $\alpha_{0}$ is locally identified) is $\left\{\alpha=\left(\alpha_{1}, \alpha_{2}, \ldots\right):\left|\alpha_{j}\right|<L^{-1},(j=1,2, \ldots)\right\}$.

It is important to note that Theorems 1 and 2 provide sufficient, and not necessary, conditions for local identification. In fact, the conditions of Theorems 1 and 2 are sufficient for

$$
\left\|m(\alpha)-m^{\prime}\left(\alpha-\alpha_{0}\right)\right\|_{\mathcal{B}} \neq\left\|m^{\prime}\left(\alpha-\alpha_{0}\right)\right\|_{\mathcal{B}}
$$

that implies $m(\alpha) \neq 0$, to hold on $\mathcal{N}$. The set where (2.2) holds may be larger than the set $\mathcal{N}$ of Theorem 1 or 2 . We have focused on the set $\mathcal{N}$ of Theorem 1 or 2 because those conditions and the associated locally identified set $\mathcal{N}$ are relatively easy to interpret. See Appendix E for more identification results related to (2.2).

Assumption 1 may not be needed for identification in nonlinear models, although local identification is complicated in the absence of Assumption 1. Conditions may involve nonzero higher order derivatives. Such results for parametric models are discussed by, e.g., Sargan (1983). Here we focus on models where Assumption 1 is satisfied.

\section{Local Identification in Hilbert Spaces}

\subsection{Full Rank Condition in Hilbert Spaces}

The restrictions imposed on $\alpha$ in Theorem 2 are not very transparent. In Hilbert spaces it is possible to give more interpretable conditions based on a lower bound for $\left\|m^{\prime}\left(\alpha-\alpha_{0}\right)\right\|_{\mathcal{B}}^{2}$. Let $\langle\cdot, \cdot\rangle$ denote the inner product for a Hilbert space. 
Assumption 3: $\left(\mathcal{A},\|\cdot\|_{\mathcal{A}}\right)$ and $\left(\mathcal{B},\|\cdot\|_{\mathcal{B}}\right)$ are separable Hilbert spaces and either a) there is a set $\mathcal{N}^{\prime}$, an orthonormal basis $\left\{\phi_{1}, \phi_{2}, \ldots\right\} \subseteq \mathcal{A}$, and a bounded, positive sequence $\left(\mu_{1}, \mu_{2}, \ldots\right)$ such that for all $\alpha \in \mathcal{N}^{\prime}$,

$$
\left\|m^{\prime}\left(\alpha-\alpha_{0}\right)\right\|_{\mathcal{B}}^{2} \geq \sum_{j=1}^{\infty} \mu_{j}^{2}\left\langle\alpha-\alpha_{0}, \phi_{j}\right\rangle^{2}
$$

or b) $m^{\prime}$ is a compact linear operator with positive singular values $\left(\mu_{1}, \mu_{2}, \ldots\right)$.

The hypothesis in b) that $m^{\prime}$ is a compact operator is a mild one when $m^{\prime}$ is a conditional expectation. Recall that an operator $m: \mathcal{A} \mapsto \mathcal{B}$ is compact if and only if it is continuous and maps bounded sets in $\mathcal{A}$ into relatively compact sets in $\mathcal{B}$. Under very mild conditions, $m(\alpha)=E[\alpha(X) \mid W]$ is compact: See Zimmer (1990, chapter 3), Kress (1999, section 2.4) and Carrasco, Florens, and Renault (2007) for a variety of sufficient conditions. When $m^{\prime}$ in b) is compact there is an orthonormal basis $\left\{\phi_{j}: j=1, \ldots\right\}$ for $\mathcal{A}$ with

$$
\left\|m^{\prime}\left(\alpha-\alpha_{0}\right)\right\|_{\mathcal{B}}^{2}=\sum_{j=1}^{\infty} \mu_{j}^{2}\left\langle\alpha-\alpha_{0}, \phi_{j}\right\rangle^{2}
$$

where $\mu_{j}^{2}$ are the eigenvalues and $\phi_{j}$ the eigenfunctions of the operator $m^{* *} m^{\prime}$, so that condition a) is satisfied, where $m^{\prime *}$ denotes the adjoint of $m^{\prime}$. The assumption that the singular values are all positive implies the rank condition holds for $\mathcal{N}^{\prime}=\mathcal{A}$. Part a) differs from part b) by imposing a lower bound on $\left\|m^{\prime}\left(\alpha-\alpha_{0}\right)\right\|_{\mathcal{B}}^{2}$ only over a subset $\mathcal{N}^{\prime}$ of $\mathcal{A}$ and by allowing the basis $\left\{\phi_{j}\right\}$ to be different from the eigenfunction basis of the operator $m^{\prime *} m^{\prime}$. In principle this allows us to impose restrictions on $\alpha-\alpha_{0}$, like boundedness and smoothness, which could help Assumption 3 a) to hold. For similar assumptions in estimation context, see, e.g., Chen and Reiß (2011) and Chen and Pouzo (2012).

It turns out that there is a precise sense in which the rank condition is satisfied for most data generating processes, if it is satisfied for one, in the Hilbert space environment here. In this sense the rank condition turns out to be generic. Let $\mathcal{A}$ and $\mathcal{B}$ be separable Hilbert spaces, and $\mathcal{N}^{\prime} \subseteq \mathcal{A}$. Suppose that there exists at least one compact linear operator: $\mathcal{K}: \mathcal{A} \mapsto \mathcal{B}$ which is injective, i.e. $\mathcal{K} \delta=0$ for $\delta \in \mathcal{A}$ if and only if $\delta=0$. This is an infinite-dimensional analog of the order condition, that for example rules out $\mathcal{B}$ having smaller finite dimension than $\mathcal{A}$ 
(e.g. having fewer instruments than right-hand side endogenous variables in a linear regression model). The operator $m^{\prime}: \mathcal{N}^{\prime} \mapsto \mathcal{B}$ is generated by the nature as follows:

1. The nature selects a countable orthonormal basis $\left\{\phi_{j}\right\}$ of cardinality $N \leq \infty$ in $\mathcal{A}$ and an orthonormal set $\left\{\varphi_{j}\right\}$ of equal cardinality in $\mathcal{B}$.

2. The nature samples a bounded sequence of real numbers $\left\{\lambda_{j}\right\}$ according to a probability measure $\eta$ whose each marginal is dominated by the Lebesgue measure on $\mathbb{R}$, namely $\operatorname{Leb}(A)=0$ implies $\eta\left(\left\{\lambda_{j} \in A\right\}\right)=0$ for any measurable $A \subset \mathbb{R}$ for each $j$.

Then the nature sets, for some scalar number $\kappa>0$, and every $\delta \in \mathcal{N}^{\prime}$

$$
m^{\prime} \delta=\kappa\left(\sum_{j=0}^{N} \lambda_{j}\left\langle\phi_{j}, \delta\right\rangle \varphi_{j}\right) .
$$

This operator is properly defined on $\mathcal{N}^{\prime}:=\left\{\delta \in \mathcal{A}: m^{\prime} \delta \in \mathcal{B}\right\}$.

LEMMA 3 (1) In the absence of further restrictions on $\mathrm{m}^{\prime}$, the algorithms obeying conditions 1 and 2 exist. (2) If $m^{\prime}$ is generated by any algorithm that obeys conditions 1 and 2, then probability that $m^{\prime}$ is not injective over $\mathcal{N}^{\prime}$ is zero, namely $\operatorname{Pr}_{\eta}\left\{\exists \delta \in \mathcal{N}^{\prime}: \delta \neq 0\right.$ and $m^{\prime} \delta=$ $0\}=0$. Moreover, Assumption 3 holds with $\mu_{j}=\left|\kappa \lambda_{j}\right|$ with probability one under $\eta$.

In Appendix B we provide examples for the case $\mathcal{A}=\mathcal{B}=L^{2}[0,1]$ that highlight the range of algorithms permitted by conditions 1 and 2 above, including cases where various restrictions on $m^{\prime}$ are imposed: boundedness, compactness, weak positivity, and density restrictions. Genericity arguments use the idea of randomization, and are often employed in economic theory, functional analysis, and probability theory, see, e.g., Anderson and Zame (2000), Marcus and Pisier (1981), Ledoux and Talagrand (2011). Andrews (2011) previously used a related notion of genericity, called prevalence within bounded sets, to argue that rich classes of operators induced by densities in nonparametric IV are $L^{2}$-complete. Though inspired in part by Andrews (2011), the simple result above uses a somewhat different notion of genericity than prevalence. 1

\footnotetext{
${ }^{1}$ Informally speaking, prevalence requires that it should be possible to construct a randomization device such that all finite-dimensional distributions for $\lambda_{j}$ 's are absolutely continuous, i.e., the distribution of $\left(\lambda_{j_{1}}, \ldots, \lambda_{j_{k}}\right)$ needs to be continuous with respect to the Lebesgue measure on $\mathbb{R}^{k}$, for any $\left(j_{1}, \ldots, j_{k}\right) \subset\{1,2, \ldots, N\}$, and any $k \in\{1,2, \ldots\}$. The notion that we use requires only that the one-dimensional marginal distributions for $\lambda_{j}$ are absolutely continuous for any $j$. The distinction is actually important to cover cases, where perfect dependence between some $\lambda_{j}$ 's may be required to maintain conditions imposed on the operator, such as, for example, the kernel of the operator being a conditional density. See Appendix B for details.
} 
We also note that while this construction implies identification with probability one, it does not regulate in any way the strength of identification, and hence has no bearing on the choice of an inferential method.

\subsection{Local Identification in Hilbert Spaces}

In what follows let $b_{j}=\left\langle\alpha-\alpha_{0}, \phi_{j}\right\rangle, j=1,2, \ldots$ denote the Fourier coefficients for $\alpha-\alpha_{0}$, so that $\alpha=\alpha_{0}+\sum_{j=1}^{\infty} b_{j} \phi_{j}$. Under Assumptions 2 and 3 we can characterize an identified set in terms of the Fourier coefficients.

Theorem 4: If Assumptions 2 and 3 are satisfied then $\alpha_{0}$ is locally identified on $\mathcal{N}=\mathcal{N}^{\prime \prime} \cap$ $\mathcal{N}^{\prime \prime \prime}$, where

$$
\mathcal{N}^{\prime \prime \prime}=\left\{\alpha=\alpha_{0}+\sum_{j=1}^{\infty} b_{j} \phi_{j}: \sum_{j=1}^{\infty} \mu_{j}^{2} b_{j}^{2}>L^{2}\left(\sum_{j=1}^{\infty} b_{j}^{2}\right)^{r}\right\}
$$

When $r=1$ it is necessary for $\alpha \in \mathcal{N}^{\prime \prime \prime}$ that the Fourier coefficients $b_{j}$ where $\mu_{j}^{2}$ is small not be too large relative to the Fourier coefficients where $\mu_{j}^{2}$ is large. In particular, when $r=1$ any $\alpha \neq \alpha_{0}$ with $b_{j}=0$ for all $j$ with $\mu_{j}>L$ will not be an element of $\mathcal{N}^{\prime \prime \prime}$. When $r>1$ we can use the Hölder inequality to obtain a sufficient condition for $\alpha \in \mathcal{N}^{\prime \prime \prime}$ that is easier to interpret.

Corollary 5: If Assumptions 2 and 3 are satisfied, with $L>0, r>1$, then $\alpha_{0}$ is locally identified on $\mathcal{N}=\mathcal{N}^{\prime \prime} \cap \mathcal{N}^{\prime \prime \prime}$ where $\mathcal{N}^{\prime \prime \prime}=\left\{\alpha=\alpha_{0}+\sum_{j=1}^{\infty} b_{j} \phi_{j}: \sum_{j=1}^{\infty} \mu_{j}^{-2 /(r-1)} b_{j}^{2}<L^{-2 /(r-1)}\right\}$.

For $\alpha$ to be in the $\mathcal{N}^{\prime \prime \prime}$ of Corollary 5 the Fourier coefficients $b_{j}$ must vanish faster than

$\mu_{j}^{1 /(r-1)}$ as $j$ grows. In particular, a sufficient condition for $\alpha \in \mathcal{N}^{\prime \prime \prime}$ is that $\left|b_{j}\right|<\left(\mu_{j} / L\right)^{1 /(r-1)} c_{j}$ for any positive sequence $c_{j}$ with $\sum_{j=1}^{\infty} c_{j}^{2}=1$. These bounds on $b_{j}$ correspond to a hyperrectangle while the $\mathcal{N}^{\prime \prime \prime}$ in Corollary 5 corresponds to an ellipsoid. The bounds on $b_{j}$ shrink as $L$ increases, corresponding to a smaller local identification set when more nonlinearity is allowed. Also, it is well known that, at least in certain environments, imposing bounds on Fourier coefficients corresponds to imposing smoothness conditions, like existence of derivatives; see for example Kress (Chapter 8, 1999). In that sense the identification set in Corollary 5 imposes smoothness conditions on the deviations of $\alpha$ from the truth $\alpha_{0}$. 
The bound imposed in $\mathcal{N}^{\prime \prime \prime}$ of Corollary 5 is a "source condition" under Assumption 3 b) and is similar to conditions used by Florens, Johannes and Van Bellegem (2011) and others. Under Assumption 3 a) it is similar to norms in generalized Hilbert scales, for example, see Engl, Hanke, and Neubauer (1996) and Chen and Reiß (2011). Our Assumption 3 a) or 3 b) are imposed on deviations $\alpha-\alpha_{0}$, while the above references all impose on true function $\alpha_{0}$ itself as well as on the parameter space hence on the deviations.

\subsection{A Quantile IV Example}

To illustrate the results of this Section we consider an endogenous quantile example where $0<\tau<1$ is a scalar,

$$
\rho(Y, X, \alpha)=1(Y \leq \alpha(X))-\tau
$$

$\mathcal{A}=\left\{\alpha(\cdot): \mathrm{E}\left[\alpha(X)^{2}\right]<\infty\right\}$, and $\mathcal{B}=\left\{a(\cdot): \mathrm{E}\left[a(W)^{2}\right]<\infty\right\}$, with the usual Hilbert spaces of mean-square integrable random variables. Here we have

$$
m(\alpha)=\mathrm{E}[1(Y \leq \alpha(X)) \mid W]-\tau
$$

Let $f_{Y}(y \mid X, W)$ denote the conditional probability density function (pdf) of $Y$ given $X$ and $W$, $f_{X}(x \mid W)$ the conditional pdf of $X$ given $W$, and $f(x)$ the marginal pdf of $X$.

Theorem 6: If $f_{Y}(y \mid X, W)$ is continuously differentiable in y with $\left|d f_{Y}(y \mid X, W) / d y\right| \leq L_{1}$, $f_{X}(x \mid W) \leq L_{2} f(x)$, and $m^{\prime} h=\mathrm{E}\left[f_{Y}\left(\alpha_{0}(X) \mid X, W\right) h(X) \mid W\right]$ satisfies Assumption 3, then $\alpha_{0}$ is locally identified on

$$
\mathcal{N}=\left\{\alpha=\alpha_{0}+\sum_{j=1}^{\infty} b_{j} \phi_{j} \in \mathcal{A}: \sum_{j=1}^{\infty} b_{j}^{2} / \mu_{j}^{2}<\left(L_{1} L_{2}\right)^{-2}\right\}
$$

This result gives a precise link between a neighborhood on which $\alpha_{0}$ is locally identified and the bounds $L_{1}$ and $L_{2}$. Assumption $3 \mathrm{~b}$ ) will hold under primitive conditions for $m^{\prime}$ to be complete, that are given by Chernozhukov, Imbens, and Newey (2007). Theorem 6 corrects Theorem 3.2 of Chernozhukov, Imbens, and Newey (2007) by adding the bound on $\sum_{j=1}^{\infty} b_{j}^{2} / \mu_{j}^{2}$. It also gives primitive conditions for local identification for general $X$ while Chernozhukov and Hansen (2005) only gave primitive conditions for identification when $X$ is discrete. Horowitz and 
Lee (2007) impose analogous conditions in their paper on convergence rates of nonparametric endogenous quantile estimators but assumed identification.

\section{Semiparametric Models}

\subsection{Identification Results}

In this section, we consider local identification in possibly nonlinear semiparametric models, where $\alpha$ can be decomposed into a $p \times 1$ dimensional parameter vector $\beta$ and nonparametric component $g$, so that $\alpha=(\beta, g)$. Let $|\cdot|$ denote the Euclidean norm for $\beta$ and assume $g \in \mathcal{G}$ where $\mathcal{G}$ is a Banach space with norm $\|\cdot\|_{\mathcal{G}}$, such as a Hilbert space. We focus here on a conditional moment restriction model

$$
\mathrm{E}\left[\rho\left(Y, X, \beta_{0}, g_{0}\right) \mid W\right]=0
$$

where $\rho(y, x, \beta, g)$ is a $J \times 1$ vector of residuals. Here $m(\alpha)=\mathrm{E}[\rho(Y, X, \beta, g) \mid W]$ will be considered as an element of the Hilbert space $\mathcal{B}$ of $J \times 1$ random vectors with inner product

$$
\langle a, b\rangle=\mathrm{E}\left[a(W)^{T} b(W)\right] .
$$

The differential $m^{\prime}\left(\alpha-\alpha_{0}\right)$ can be expressed as

$$
m^{\prime}\left(\alpha-\alpha_{0}\right)=m_{\beta}^{\prime}\left(\beta-\beta_{0}\right)+m_{g}^{\prime}\left(g-g_{0}\right),
$$

where $m_{\beta}^{\prime}$ is the derivative of $m\left(\beta, g_{0}\right)=\mathrm{E}\left[\rho\left(Y, X, \beta, g_{0}\right) \mid W\right]$ with respect to $\beta$ at $\beta_{0}$ and $m_{g}^{\prime}$ is the Gâteaux derivative of $m\left(\beta_{0}, g\right)$ with respect to $g$ at $g_{0}$. To give conditions for local identification of $\beta_{0}$ in the presence of the nonparametric component $g$ it is helpful to partial

out $g$. Let $\overline{\mathcal{M}}$ be the closure of the linear span $\mathcal{M}$ of $m_{g}^{\prime}\left(g-g_{0}\right)$ for $g \in \mathcal{N}_{g}^{\prime}$ where $\mathcal{N}_{g}^{\prime}$ will be specified below. In general $\overline{\mathcal{M}} \neq \mathcal{M}$ because the linear operator $m_{g}^{\prime}$ need not have closed range (like $m^{\prime}$ onto, a closed range would also imply a continuous inverse, by the Banach inverse theorem). For the $k^{\text {th }}$ unit vector $e_{k},(k=1, \ldots, p)$, let

$$
\zeta_{k}^{*}=\arg \min _{\zeta \in \overline{\mathcal{M}}} \mathrm{E}\left[\left\{m_{\beta}^{\prime}(W) e_{k}-\zeta(W)\right\}^{T}\left\{m_{\beta}^{\prime}(W) e_{k}-\zeta(W)\right\}\right],
$$

which exists and is unique by standard Hilbert space results; e.g. see Luenberger (1969). Define $\Pi$ to be the $p \times p$ matrix with

$$
\Pi_{j k}:=\mathrm{E}\left[\left\{m_{\beta}^{\prime}(W) e_{j}-\zeta_{j}^{*}(W)\right\}^{T}\left\{m_{\beta}^{\prime}(W) e_{k}-\zeta_{k}^{*}(W)\right\}\right], \quad(j, k=1, \ldots, p) .
$$


The following condition is important for local identification of $\beta_{0}$.

Assumption 4: $m^{\prime}: \mathbb{R}^{p} \times \mathcal{N}_{g}^{\prime} \longrightarrow \mathcal{B}$ is linear and bounded, and $\Pi$ is nonsingular.

This assumption is similar to those first used by Chamberlain (1992) to establish the possibility of estimating parametric components at root- $n$ rate in semi-parametric moment condition problems; see also Ai and Chen (2003) and Chen and Pouzo (2009). In the local identification analysis considered here it leads to local identification of $\beta_{0}$ without identification of $g$ when $m\left(\beta_{0}, g\right)$ is linear in $g$. It allows us to separate conditions for identification of $\beta_{0}$ from conditions for identification of $g$. Note that the parameter $\beta$ may be identified even when $\Pi$ is singular, but that case is more complicated, as discussed at the end of Section 2, and we do not analyze this case.

The following condition controls the behavior of the derivative with respect to $\beta$ :

Assumption 5: For every $\varepsilon>0$ there is a neighborhood $B$ of $\beta_{0}$ and a set $\mathcal{N}_{g}^{\beta}$ such that for all $g \in \mathcal{N}_{g}^{\beta}$ with probability one $\mathrm{E}[\rho(Y, X, \beta, g) \mid W]$ is continuously differentiable in $\beta$ on $B$ and

$$
\sup _{g \in \mathcal{N}_{g}^{\beta}} \sqrt{\mathrm{E}\left[\sup _{\beta \in B}\left|\partial \mathrm{E}[\rho(Y, X, \beta, g) \mid W] / \partial \beta-\partial \mathrm{E}\left[\rho\left(Y, X, \beta_{0}, g_{0}\right) \mid W\right] / \partial \beta\right|^{2}\right]}<\varepsilon .
$$

It turns out that Assumptions 4 and 5 will be sufficient for local identification of $\beta_{0}$ when $m\left(\beta_{0}, g\right)$ is linear in $g$, i.e. for $m(\beta, g)=0$ to imply $\beta=\beta_{0}$ when $(\beta, g)$ is in some neighborhood of $\left(\beta_{0}, g_{0}\right)$. This works because Assumption 4 partials out the effect of unknown $g$ on local identification of $\beta_{0}$.

Theorem 7: If Assumptions 4 and 5 are satisfied and $m\left(\beta_{0}, g\right)$ is linear in $g$ then there is an $\varepsilon>0$ such that for $B$ and $\mathcal{N}_{g}^{\beta}$ from Assumption 5 and $\mathcal{N}_{g}^{\prime}$ from Assumption $4, \beta_{0}$ is locally identified for $\mathcal{N}=B \times\left(\mathcal{N}_{g}^{\prime} \cap \mathcal{N}_{g}^{\beta}\right)$. If, in addition, Assumption 1 is satisfied for $m_{g}^{\prime}$ and $\mathcal{N}_{g}^{\prime} \cap \mathcal{N}_{g}^{\beta}$ replacing $m^{\prime}$ and $\mathcal{N}^{\prime}$ then $\alpha_{0}=\left(\beta_{0}, g_{0}\right)$ is locally identified for $\mathcal{N}$.

This result is more general than Florens, Johannes, and Van Bellegem (2012) and Santos (2011) since it allows for nonlinearities in $\beta$, and dependence on $g$ of the partial derivatives $\partial \mathrm{E}[\rho(Y, X, \beta, g) \mid W] / \partial \beta$. When the partial derivatives $\partial \mathrm{E}[\rho(Y, X, \beta, g) \mid W] / \partial \beta$ do not depend 
on $g$, then Assumption 5 could be satisfied with $\mathcal{N}_{g}^{\prime}=\mathcal{G}$, and Theorem 7 could then imply local identification of $\beta_{0}$ in some neighborhood of $\beta_{0}$ only.

For semiparametric models that are nonlinear in $g$ we can give local identification results based on Theorem 2 or the more specific conditions of Theorem 4 and Corollary 5. For brevity we give just a result based on Theorem 2 .

TheOrem 8: If Assumptions 4 and 5 are satisfied and $m\left(\beta_{0}, g\right)$ satisfies Assumption 2 with $\mathcal{N}^{\prime \prime}=\mathcal{N}_{g}^{\prime \prime}$, then there is an $\varepsilon>0$ such that for $B$ and $\mathcal{N}_{g}^{\beta}$ from Assumption $5, \mathcal{N}_{g}^{\prime}$ from Assumption 4, and

$$
\mathcal{N}_{g}^{\prime \prime \prime}=\left\{g:\left\|m_{g}^{\prime}\left(g-g_{0}\right)\right\|_{\mathcal{B}}>\varepsilon^{-1} L\left\|g-g_{0}\right\|_{\mathcal{A}}^{r}\right\}
$$

it is the case that $\alpha_{0}=\left(\beta_{0}, g_{0}\right)$ is locally identified for $\mathcal{N}=B \times\left(\mathcal{N}_{g}^{\beta} \cap \mathcal{N}_{g}^{\prime} \cap \mathcal{N}_{g}^{\prime \prime} \cap \mathcal{N}_{g}^{\prime \prime \prime}\right)$.

\subsection{A Single Index IV Example}

Econometric applications often have too many covariates for fully nonparametric estimation to be practical, i.e. they suffer from the curse of dimensionality. Econometric practice thus motivates interest in models with reduced dimension. An important such model is the single index model. Here we consider a single index model with endogeneity, given by

$$
Y=g_{0}\left(X_{1}+X_{2}^{T} \beta_{0}\right)+U, \quad \mathrm{E}[U \mid W]=0
$$

where $\beta_{0}$ is a vector of unknown parameters, $g_{0}(\cdot)$ is an unknown function, and $W$ are instrumental variables. Here the nonparametric part is just one dimensional rather than having the same dimension as $X$. This model is nonlinear in Euclidean parameters, and so is an example where our results apply. Our results add to the literature on dimension reduction with endogeneity, by showing how identification of an index model requires fewer instrumental variables than a fully nonparametric IV model. We could generalize the results to multiple indices but focus on a single index for simplicity.

The location and scale of the parametric part are not identified separately from $g_{0}$, and hence, we normalize the constant to zero and the coefficient of $X_{1}$ to 1 . Here

$$
m(\alpha)(W)=\mathrm{E}\left[Y-g\left(X_{1}+X_{2}^{T} \beta\right) \mid W\right]
$$


Let $V=X_{1}+X_{2}^{T} \beta_{0}$ and for differentiable $g_{0}(V)$ let

$$
m_{\beta}^{\prime}=-\mathrm{E}\left[g_{0}^{\prime}(V) X_{2}^{T} \mid W\right]
$$

Let $\zeta_{j}^{*}$ denote the projection of $m_{\beta}^{\prime} e_{j}=-\mathrm{E}\left[g_{0}^{\prime}(V) X_{2 j} \mid W\right]$ on the mean-square closure of the set $\left\{\mathrm{E}[h(V) \mid W]: \mathrm{E}\left[h(V)^{2}\right]<\infty\right\}$ and $\Pi$ the matrix with $\Pi_{j k}=\mathrm{E}\left[\left(m_{\beta}^{\prime} e_{j}-\zeta_{j}^{*}\right)\left(m_{\beta}^{\prime} e_{k}-\zeta_{k}^{*}\right)\right]$.

TheOrem 9: Consider the model of equation (4.4). If a) $g_{0}(V)$ is continuously differentiable with bounded derivative $g_{0}^{\prime}(V)$ satisfying $\left|g_{0}^{\prime}(\tilde{V})-g_{0}^{\prime}(V)\right| \leq C_{g}|\tilde{V}-V|$ for some $C_{g}>0$, b) $\mathrm{E}\left[\left|X_{2}\right|^{4}\right]<\infty$, and c) $\Pi$ is nonsingular, then there is a neighborhood $B$ of $\beta_{0}$ and $\delta>0$ such that for

$$
\mathcal{N}_{g}^{\delta}=\left\{g: g(v) \text { is continuously differentiable and } \sup _{v}\left|g^{\prime}(v)-g_{0}^{\prime}(v)\right| \leq \delta\right\}
$$

$\beta_{0}$ is locally identified for $\mathcal{N}=B \times \mathcal{N}_{g}^{\delta}$. Furthermore, if there is $\mathcal{N}_{g}^{\prime}$ such that $\mathrm{E}\left[g(V)-g_{0}(V) \mid W\right]$ is bounded complete on the set $\left\{g(V)-g_{0}(V): g \in \mathcal{N}_{g}^{\prime}\right\}$ then $\left(\beta_{0}, g_{0}\right)$ is locally identified for $\mathcal{N}=B \times\left(\mathcal{N}_{g}^{\delta} \cap \mathcal{N}_{g}^{\prime}\right)$.

Since this model includes as a special case the linear simultaneous equations model the usual rank and order conditions are still necessary for $\Pi$ to be nonsingular for all possible models, and hence are necessary for identification. Relative to the linear nonparametric IV model in Newey and Powell (2003) the index structure lowers the requirements for identification by requiring that $m_{g}^{\prime} h=-\mathrm{E}[h(V) \mid W]$ be complete on $\mathcal{N}_{g}^{\prime}$ rather than completeness of the conditional expectation of functions of $X$ given $W$. For example, it may be possible to identify $\beta_{0}$ and $g_{0}$ with only two instrumental variables, one of which is used to identify $g_{0}$ and functions of the other being used to identify $\beta_{0}$.

To further explain we can give more primitive conditions for nonsingularity of $\Pi$. The following result gives a necessary condition for $\Pi$ to be nonzero (and hence nonsingular) as well as a sufficient condition for nonsingularity of $\Pi$.

TheOrem 10: Consider the model of 4.4). If $\Pi$ is nonsingular then the conditional distribution of $W$ given $V$ is not complete. Also, if there is a measurable function $T(W)$ such that 
the conditional distribution of $V$ given $W$ depends only on $T(W)$ and for every $p \times 1$ vector $\lambda \neq 0, \mathrm{E}\left[g_{0}^{\prime}(V) \lambda^{T} X_{2} \mid W\right]$ is not measurable with respect to $T(W)$, then $\Pi$ is nonsingular.

To explain the conditions of this result note that if there is only one variable in $W$ then the completeness condition (of $W$ given $V$ ) can hold and hence $\Pi$ can be singular. If there is more than one variable in $W$ then generally completeness (of $W$ given $V$ ) will not hold, because completeness would be like identifying a function of more than one variable (i.e. $W$ ) with one instrument (i.e. $V$ ). If $W$ and $V$ are joint Gaussian and $V$ and $W$ are correlated then completeness holds (and hence $\Pi$ is singular) when $W$ is one dimensional but not otherwise. In this sense having more than one instrument in $W$ is a necessary condition for nonsingularity of $\Pi$. Intuitively, one instrument is needed for identification of the one dimensional function $g_{0}(V)$ so that more than one instrument is needed for identification of $\beta$.

The sufficient condition for nonsingularity of $\Pi$ is stronger than noncompleteness. It is essentially an exclusion restriction, where $\mathrm{E}\left[g_{0}^{\prime}(V) X_{2} \mid W\right]$ depends on $W$ in a different way than the conditional distribution of $V$ depends on $W$. This condition can be shown to hold if $W$ and $V$ are Gaussian, $W$ is two dimensional, and $\mathrm{E}\left[g_{0}^{\prime}(V) X_{2} \mid W\right]$ depends on all of $W$.

\section{Semiparametric CCAPM}

Consumption capital asset pricing models (CCAPM) provide interesting examples of nonparametric and semiparametric moment restrictions; see Gallant and Tauchen (1989), Newey and Powell (1988), Hansen, Heaton, Lee, and Roussanov (2007), Chen and Ludvigson (2009), and others. In this section, we apply our general theorems to develop new results on identification of a particular semiparametric specification of marginal utility of consumption. Our results could easily be extended to other specifications, and so are of independent interest.

To describe the model let $C_{t}$ denote consumption level at time $t$ and $c_{t} \equiv C_{t} / C_{t-1}$ be consumption growth. Suppose that the marginal utility of consumption at time $t$ is given by

$$
M U_{t}=C_{t}^{-\gamma_{0}} g_{0}\left(C_{t} / C_{t-1}\right)=C_{t}^{-\gamma_{0}} g_{0}\left(c_{t}\right)
$$

where $g_{0}(c)$ is an unknown positive function. For this model the intertemporal marginal rate 
of substitution is

$$
\delta_{0} M U_{t+1} / M U_{t}=\delta_{0} c_{t+1}^{-\gamma_{0}} g_{0}\left(c_{t+1}\right) / g_{0}\left(c_{t}\right),
$$

where $0<\delta_{0} \leq 1$ is the rate of time preference. Let $R_{t+1}=\left(R_{t+1,1}, \ldots, R_{t+1, J}\right)^{T}$ be a $J \times 1$ vector of gross asset returns. A semiparametric CCAPM equation is then given by

$$
\mathrm{E}\left[R_{t+1} \delta_{0} c_{t+1}^{-\gamma_{0}}\left\{g_{0}\left(c_{t+1}\right) / g_{0}\left(c_{t}\right)\right\} \mid W_{t}\right]=e,
$$

where $e$ is a $J \times 1$ vector of ones, and $W_{t} \equiv\left(Z_{t}^{T}, c_{t}\right)^{T}$ is a vector of random variables observed by the agent at time $t$, with $Z_{t}$ not a measurable function of $c_{t}$. This corresponds to an external habit formation model with only one lag, a special case of Chen and Ludvigson (2009). As emphasized in Cochrane (2005), habit formation models can help explain the high risk premia embedded in asset prices. We focus here on consumption growth $c_{t}=C_{t} / C_{t-1}$ to circumvent the potential nonstationarity of the level of consumption, see Hall (1978), as has long been done in this literature, e.g. Hansen and Singleton (1982).

From economic theory it is known that under complete markets there is a unique intertemporal marginal rate of substitution that solves equation (5.5), when $R_{t}$ is allowed to vary over all possible vectors of asset returns. Of course that does not guarantee a unique solution for a fixed vector of returns $R_{t}$. Note though that the semiparametric model does impose restrictions on the marginal rate of substitution that should be helpful for identification. We show how these restrictions lead to local identification of this model via the results of Section 4.

This model can be formulated as a semiparametric conditional moment restriction by letting $Y=\left(R_{t+1}^{T}, c_{t+1}, c_{t}\right)^{T}, \beta=(\delta, \gamma)^{T}, W=W_{t}=\left(Z_{t}^{T}, c_{t}\right)^{T}$, and

$$
\rho(Y, \beta, g)=R_{t+1} \delta c_{t+1}^{-\gamma} g\left(c_{t+1}\right)-g\left(c_{t}\right) e
$$

Then multiplying equation (5.5) through by $g_{0}\left(c_{t}\right)$ gives the conditional moment restriction $\mathrm{E}\left[\rho\left(Y, \beta_{0}, g_{0}\right) \mid W\right]=0$. Let $A_{t}=R_{t+1} \delta_{0} c_{t+1}^{-\gamma_{0}}$. The nonparametric rank condition (Assumption 1 for $g$ ) will be uniqueness, up to scale, of the solution $g_{0}$ of

$$
\mathrm{E}\left[A_{t} g\left(c_{t+1}\right) \mid W_{t}\right]=g\left(c_{t}\right) e
$$

This equation differs from the linear nonparametric IV restriction where the function $g_{0}(X)$ would solve $\mathrm{E}[Y \mid W]=\mathrm{E}[g(X) \mid W]$. That equation is an integral equation of the first kind while 
equation (5.7) is a homogeneous integral equation of the second kind. The rank condition for this second kind equation is that the null space of the operator $\mathrm{E}\left[A_{t} g\left(c_{t+1}\right) \mid W_{t}\right]-g\left(c_{t}\right) e$ is onedimensional, which is different than the completeness condition for first kind equations. This example illustrates that the rank condition of Assumption 1 need not be equivalent to completeness of a conditional expectation. Escanciano and Hoderlein (2010) and Lewbel, Linton, and Srisuma (2012) have previously shown how homogenous integral equations of the second kind arise in CCAPM models, though their models and identification results are different than those given here, as further discussed below.

Let $X_{t}=\left(1 / \delta_{0},-\ln \left(c_{t+1}\right)\right)^{T}$. Then differentiating inside the integral, as allowed under regularity conditions given below, and applying the Gateaux derivative calculation gives

$$
m_{\beta}^{\prime}(W)=\mathrm{E}\left[A_{t} g_{0}\left(c_{t+1}\right) X_{t}^{T} \mid W_{t}\right], \quad m_{g}^{\prime} g=\mathrm{E}\left[A_{t} g\left(c_{t+1}\right) \mid W_{t}\right]-g\left(c_{t}\right) e .
$$

When $\mathrm{E}\left[A_{t} g\left(c_{t+1}\right) \mid W_{t}\right]$ is a compact operator, as holds under conditions described below, it follows from the theory of integral equations of the second kind (e.g. Kress, 1999, Theorem 3.2) that the set of nonparametric directions $\mathcal{M}$ will be closed, i.e.

$$
\overline{\mathcal{M}}=\mathcal{M}=\left\{\mathrm{E}\left[A_{t} g\left(c_{t+1}\right) \mid W_{t}\right]-g\left(c_{t}\right) e:\|g\|_{\mathcal{G}}<\infty\right\}
$$

where we will specify $\|g\|_{\mathcal{G}}$ below. Let $\Pi$ be the two-dimensional second moment matrix $\Pi$ of the residuals from the projection of each column of $m_{\beta}^{\prime}$ on $\overline{\mathcal{M}}$, as described in Section 4 . Then nonsingularity of $\Pi$ leads to local identification of $\beta_{0}$ via Theorem 7 .

To give a precise result let $\Delta$ be any finite positive number,

$$
\begin{aligned}
D_{t} & =\left(1+\left|R_{t+1}\right|\right)\left[2+\left|\ln \left(c_{t+1}\right)\right|^{2}\right] \sup _{\gamma \in\left[\gamma_{0}-\Delta, \gamma_{0}+\Delta\right]} c_{t+1}^{-\gamma}, \\
\mathcal{G} & =\left\{g:\|g\|_{\mathcal{G}} \equiv \sqrt{\mathrm{E}\left[E\left[D_{t}^{2} \mid W_{t}\right] g\left(c_{t+1}\right)^{2}\right]}<\infty\right\} .
\end{aligned}
$$

The following assumption imposes some regularity conditions.

Assumption 6: $\left(R_{t}^{T}, c_{t}, Z_{t}^{T}\right)$ is strictly stationary, $\mathrm{E}\left[D_{t}^{2}\right]<\infty ; 0<\delta_{0} \leq 1,\left\|g_{0}\right\|_{\mathcal{G}}<\infty$.

The following result applies Theorem 7 to this CCAPM. 
Theorem 11: Consider equation 5.7). Suppose that Assumption 6 is satisfied. Then the linear mapping $m^{\prime}: \mathbb{R}^{2} \times \mathcal{G} \longrightarrow \mathcal{B}$ is bounded and if $\Pi$ is nonsingular there is a neighborhood $B$ of $\beta_{0}$ and $\varepsilon>0$ such that for $\mathcal{N}_{g}^{\beta}=\left\{g:\left\|g-g_{0}\right\|_{\mathcal{G}}<\varepsilon\right\}, \beta_{0}$ is locally identified for $\mathcal{N}=B \times \mathcal{N}_{g}^{\beta}$. If in addition $m_{g}^{\prime}\left(g-g_{0}\right) \neq 0$ for all $g \neq g_{0}$ and $g \in \mathcal{N}_{g}^{\beta}$ then $\left(\beta_{0}, g_{0}\right)$ is locally identified for $\mathcal{N}=B \times \mathcal{N}_{g}^{\beta}$.

Primitive conditions for nonsingularity of $\Pi$ and for $m_{g}^{\prime}\left(g-g_{0}\right) \neq 0$ when $g \neq g_{0}$ are needed to make this result interesting. It turns out that some completeness conditions suffice, as shown by the following result. Let $\tilde{W}_{t}=\left(w\left(Z_{t}\right), c_{t}\right)$ for some measurable function $w\left(Z_{t}\right)$ of $Z_{t}$, and $f_{c, \tilde{W}}(c, \tilde{w})$ denote the joint pdf of $\left(c_{t+1}, \tilde{W}_{t}\right), f_{c}(c)$ and $f_{\tilde{W}}(\tilde{w})$ the marginal pdfs of $c_{t+1}$ and $\tilde{W}_{t}$ respectively.

Theorem 12: Consider equation (5.7). Suppose that Assumption 6 is satisfied, $\operatorname{Pr}\left(g_{0}\left(c_{t}\right)=\right.$ $0)=0$, for some $w\left(Z_{t}\right)$ and $\tilde{W}_{t}=\left(w\left(Z_{t}\right), c_{t}\right),\left(c_{t+1}, \tilde{W}_{t}\right)$ is continuously distributed and there is some $j$ with $A_{t j}=\delta_{0} R_{t+1, j} c_{t+1}^{-\gamma_{0}}$ satisfying

$$
\mathrm{E}\left[A_{t j}^{2} f_{c}\left(c_{t+1}\right)^{-1} f_{\tilde{W}}\left(\tilde{W}_{t}\right)^{-1} f_{c, \tilde{W}}\left(c_{t+1}, \tilde{W}_{t}\right)\right]<\infty .
$$

Then (a) if $\mathrm{E}\left[A_{t j} \tilde{h}\left(c_{t+1}, c_{t}\right) \mid \tilde{W}_{t}\right]=0$ implies $\tilde{h}\left(c_{t+1}, c_{t}\right)=0$ a.s. and $a\left(c_{t+1}\right)+b\left(c_{t}\right)=0$ for $c_{t} \in \mathcal{C}$ with $\operatorname{Pr}(\mathcal{C})>0$ implies a $\left(c_{t+1}\right)$ is constant then $\Pi$ is nonsingular; (b) if $g_{0} \in \mathcal{G}_{\bar{c}} \equiv\{g \in$ $\mathcal{G}: g(\bar{c}) \neq 0\}$ for some $\bar{c}$ and $\mathrm{E}\left[A_{t j} h\left(c_{t+1}\right) \mid w\left(Z_{t}\right), c_{t}=\bar{c}\right]=0$ with $h \in \mathcal{G}_{\bar{c}}$ implies $h\left(c_{t+1}\right)=0$ a.s., then $g_{0}$ is the unique solution to $\mathrm{E}\left[A_{t} g\left(c_{t+1}\right) \mid W_{t}\right]=g\left(c_{t}\right)$ up to scale.

Equation (5.8) implies $\mathrm{E}\left[A_{t j} g\left(c_{t+1}\right) \mid \tilde{W}_{t}\right]$ is a Hilbert-Schmidt integral operator and hence compact. Analogous conditions could be imposed to ensure that $\mathcal{M}$ is closed. The sufficient conditions for nonsingularity of $\Pi$ involve completeness of the conditional expectation $\mathrm{E}\left[A_{t j} h\left(c_{t+1}, c_{t}\right) \mid \tilde{W}_{t}\right]$ and a stronger version of a measurably separable condition from Florens, Mouchart, and Rolin (1990). As previously noted, sufficient conditions for completeness can be found in Newey and Powell (2003) and Andrews (2011) and completeness is generic in the sense of Andrews (2011) and Lemma 3. A simple sufficient condition for the measurably separable hypothesis is that the support of $\left(c_{t+1}, c_{t}\right)$ is $\Re_{+}^{2}$, where $\Re_{+}=[0, \infty)$. 
Condition b) is weaker than condition a). Condition (b) turns out to imply global identification of $\beta_{0}$ and $g_{0}$ (up to scale) if $g_{0}(c)$ is bounded, and bounded away from zero. Because we focus on applying the results of Section 4, we reserve this result to Theorem A.3 in Appendix D. Even with global identification the result of Theorem 12 (a) is of interest, because nonsingularity of $\Pi$ will be necessary for $\gamma_{0}$ to be estimable at a root-n rate. The identification result for $\gamma_{0}$ in Theorem A.3 involves large and small values of consumption growth, and so amounts to identification at infinity, that may not lead to root-n consistent estimation, e.g. see Chamberlain (1986).

A different approach to the nonparametric rank condition, that does not require any instrument $w\left(Z_{t}\right)$ in addition to $c_{t}$, can be based on positivity of $g_{0}(c)$. The linear operator $\mathrm{E}\left[A_{t j} g\left(c_{t+1}\right) \mid c_{t}\right]$ and $g(c)$ will be infinite dimensional (functional) analogs of a positive matrix and a positive eigenvector respectively, by equation (5.7). The Perron-Frobenius Theorem says that there is a unique positive eigenvalue and eigenvector (up to scale) pair for a positive matrix. A functional analog, based on Krein and Rutman (1950), gives uniqueness of $g_{0}(c)$, as well as of the discount factor $\delta_{0}$. To describe this result let $r(c, s)=\mathrm{E}\left[R_{t+1, j} \mid c_{t+1}=\right.$ $\left.s, c_{t}=c\right], f(s, c)$ be the joint pdf of $\left(c_{t+1}, c_{t}\right), f(c)$ the marginal pdf of $c_{t}$ at $c$ and $K(c, s)=$ $r(c, s) s^{-\gamma_{0}} f(s, c) /[f(s) f(c)]$. Then the equation $\mathrm{E}\left[A_{t j} g\left(c_{t+1}\right) \mid c_{t}\right]=g\left(c_{t}\right)$ can be written as

$$
\delta \int K(c, s) g(s) f(s) d s=g(c)
$$

for $\delta=\delta_{0} \in(0,1]$. Here the matrix analogy is clear, with $K(c, s) f(s)$ being like a positive matrix, $g(c)$ an eigenvector, and $\delta^{-1}$ an eigenvalue.

Theorem 13: Suppose that $\left(R_{t, j}, c_{t}\right)$ is strictly stationary, $f(c, s)>0$ and $r(c, s)>0$ almost everywhere, and $\iint K(c, s)^{2} f(c) f(s) d c d s<\infty$. Then equation (5.9) has a unique positive solution $\left(\delta_{0}, g_{0}\right)$ in the sense that $\delta_{0}>0, g_{0}>0$ almost everywhere and $E\left[g_{0}\left(c_{t}\right)^{2}\right]=1$.

The conditions of this result include $r(c, s)>0$, that will hold if $R_{t+1, j}$ is a positive risk free rate. Under square-integrability of $K$, we obtain global identification of the pair $\left(\delta_{0}, g_{0}\right)$. The uniqueness of $g_{0}(c)$ in the conclusion of this result implies the nonparametric rank condition. Note that by iterated expectation and inclusion of $R_{t+1, j}$ in $R_{t+1}$ any solution to equation (5.7) 
must also satisfy equation (5.9). Thus Theorem 13 implies that $g_{0}$ is the unique solution to (5.7). Theorem 13 actually gives more, identification of the discount factor given identification of $\gamma_{0}$.

Previously Escanciano and Hoderlein (2010) and Lewbel, Linton and Srisuma (2012) considered nonparametric identification of marginal utility in consumption level, $M U\left(C_{t}\right)$, by solving the homogeneous integral equation of the second kind:

$$
\mathrm{E}\left[R_{t+1, j} \delta_{0} M U\left(C_{t+1}\right) \mid C_{t}\right]=M U\left(C_{t}\right) .
$$

In particular, Escanciano and Hoderlein (2010) gave an insightful identification result for the discount factor $\delta$ and the marginal utility $M U$ based on the positivity of marginal utility and a version of Perron-Frobenius theorem, but assuming that $C_{t}$ has a compact support, that $M U$ is uniformly continuous on the support, and that $R_{t+1, j}$ is a risk-free rate. Lewbel, Linton and Srisuma (2012) used a genericity argument for identification of $M U\left(C_{t}\right)$. While we also use the positivity of the unknown function $g$, we identify the "correction term" $g(c)$ in the habit-formation model, rather than the marginal utility $M U$. And we base our result on a Krein and Rutman (1950) theorem, a particular functional analog of Perron-Frobenius, which allows us to completely avoid making the compactness restriction on the support of $C_{t}$ and even of $c_{t}=C_{t+1} / C_{t} 2^{2}$ Finally, Perron-Frobenius theory has been extensively used by Hansen and Scheinkman $(2009,2012)$ and Hansen (2012) in their research on the long-run risk and dynamic valuations in a general Markov environment in which their valuation operators may not be compact. Our results follow the same general path as all of the papers cited in this paragraph, though we apply a Krein and Rutman (1950) theorem (and its extensions) to a different operator than theirs.

The models considered here will generally be highly overidentified. We have analyzed identification using only a single asset return $R_{t+1, j}$. The presence of more asset returns in equation (5.5) provides overidentifying restrictions. Also, in Theorem 12 we only use a function $w\left(Z_{t}\right)$ of the available instrumental variables $Z_{t}$ in addition to $c_{t}$. The additional information in $Z_{t}$ may provide overidentifying restrictions. These sources of overidentification are familiar in CCAPM

\footnotetext{
${ }^{2}$ The latter seems to be important to accommodate standard consumption-based asset pricing models, in which $C_{t}$ is not stationary and has a non-compact support of $(0, \infty)$.
} 
models. See, e.g., Hansen and Singleton (1982) and Chen and Ludvigson (2009).

\section{Conclusion}

We provide sufficient conditions for local identification for a general class of semiparametric and nonparametric conditional moment restriction models. We give new identification conditions for several important models that illustrate the usefulness of our general results. In particular, we provide primitive conditions for local identification in nonseparable quantile IV models, single-index IV models, and semiparametric consumption-based asset pricing models.

\section{Appendix}

\section{A Proofs for Section 2}

\section{A.1 Proof of Parametric Result}

By $\operatorname{rank}\left(m^{\prime}\right)=p$, the nonnegative square root $\eta$ of the smallest eigenvalue $\eta^{2}$ of $\left(m^{\prime}\right)^{T} m^{\prime}$ is positive and $\left|m^{\prime} h\right| \geq \eta|h|$ for $h \in \mathbb{R}^{p}$. Also, by the definition of the derivative there is $\varepsilon>0$ such that $\left|m(\alpha)-m\left(\alpha_{0}\right)-m^{\prime}\left(\alpha-\alpha_{0}\right)\right| /\left|\alpha-\alpha_{0}\right|<\eta$ for all $\left|\alpha-\alpha_{0}\right|<\varepsilon$ with $\alpha \neq \alpha_{0}$. Then

$$
\frac{\left|m(\alpha)-m^{\prime}\left(\alpha-\alpha_{0}\right)\right|}{\left|m^{\prime}\left(\alpha-\alpha_{0}\right)\right|}=\frac{\left|m(\alpha)-m\left(\alpha_{0}\right)-m^{\prime}\left(\alpha-\alpha_{0}\right)\right|}{\left|\alpha-\alpha_{0}\right|} \frac{\left|\alpha-\alpha_{0}\right|}{\left|m^{\prime}\left(\alpha-\alpha_{0}\right)\right|}<\frac{\eta}{\eta}=1 .
$$

This inequality implies $m(\alpha) \neq 0$, so $\alpha_{0}$ is locally identified on $\left\{\alpha:\left|\alpha-\alpha_{0}\right|<\varepsilon\right\}$. Q.E.D.

\section{A.2 Proof of Theorem 1}

If $m^{\prime} h=m^{\prime} \tilde{h}$ for $h \neq \tilde{h}$ then for any $\lambda>0$ we have $m^{\prime} \bar{h}=0$ for $\bar{h}=\lambda(h-\tilde{h}) \neq 0$. For $\lambda$ small enough $\bar{h}$ would be in any open ball around zero. Therefore, Assumption 1 holding on an open ball containing $\alpha_{0}$ implies that $m^{\prime}$ is invertible. By $m^{\prime}$ onto and the Banach Inverse Theorem (Luenberger, 1969, p. 149) it follows that $\left(m^{\prime}\right)^{-1}$ is continuous. Since any continuous linear map is bounded, it follows that there exists $\eta>0$ such that $\left\|m^{\prime}\left(\alpha-\alpha_{0}\right)\right\|_{\mathcal{B}} \geq \eta\left\|\alpha-\alpha_{0}\right\|_{\mathcal{A}}$ for all $\alpha \in \mathcal{A}$.

Next, by Fréchet differentiability at $\alpha_{0}$ there exists an open ball $\mathcal{N}_{\varepsilon}$ centered at $\alpha_{0}$ such that for all $\alpha \in \mathcal{N}_{\varepsilon}, \alpha \neq \alpha_{0}$,

$$
\frac{\left\|m(\alpha)-m\left(\alpha_{0}\right)-m^{\prime}\left(\alpha-\alpha_{0}\right)\right\|_{\mathcal{B}}}{\left\|\alpha-\alpha_{0}\right\|_{\mathcal{A}}}<\eta .
$$


Therefore, at all such $\alpha \neq \alpha_{0}$,

$$
\begin{aligned}
\frac{\left\|m(\alpha)-m^{\prime}\left(\alpha-\alpha_{0}\right)\right\|_{\mathcal{B}}}{\left\|m^{\prime}\left(\alpha-\alpha_{0}\right)\right\|_{\mathcal{B}}} & =\frac{\left\|m(\alpha)-m\left(\alpha_{0}\right)-m^{\prime}\left(\alpha-\alpha_{0}\right)\right\|_{\mathcal{B}}}{\left\|\alpha-\alpha_{0}\right\|_{\mathcal{A}}} \frac{\left\|\alpha-\alpha_{0}\right\|_{\mathcal{A}}}{\left\|m^{\prime}\left(\alpha-\alpha_{0}\right)\right\|_{\mathcal{B}}} \\
& <\eta / \eta=1 .
\end{aligned}
$$

Therefore, as in the proof of the parametric result above, $m(\alpha) \neq 0$ for all $\alpha \in \mathcal{N}_{\varepsilon}$ with $\alpha \neq \alpha_{0}$. Q.E.D.

\section{A.3 Proof of Theorem 2}

Consider $\alpha \in \mathcal{N}$ with $\alpha \neq \alpha_{0}$. Then

$$
\frac{\left\|m(\alpha)-m^{\prime}\left(\alpha-\alpha_{0}\right)\right\|_{\mathcal{B}}}{\left\|m^{\prime}\left(\alpha-\alpha_{0}\right)\right\|_{\mathcal{B}}}=\frac{\left\|m(\alpha)-m\left(\alpha_{0}\right)-m^{\prime}\left(\alpha-\alpha_{0}\right)\right\|_{\mathcal{B}}}{\left\|m^{\prime}\left(\alpha-\alpha_{0}\right)\right\|_{\mathcal{B}}} \leq \frac{L\left\|\alpha-\alpha_{0}\right\|_{\mathcal{A}}^{r}}{\left\|m^{\prime}\left(\alpha-\alpha_{0}\right)\right\|_{\mathcal{B}}}<1 .
$$

The conclusion follows as in the proof of Theorem 1. Q.E.D.

\section{B Proofs for Section 3}

\section{B.1 An Example for Lemma 3}

It is useful to give explicit examples of the randomization algorithms obeying conditions 1 and 2 listed in Section 3. Suppose $\mathcal{A}=\mathcal{B}=L^{2}[0,1]$, and that $m^{\prime}$ is an integral operator

$$
m^{\prime} \delta=\int K(\cdot, t) \delta(t) d t
$$

The kernel $K$ of this operator is generated as follows. The nature performs step 1 by selecting two, possibly different, orthonormal bases $\left\{\phi_{j}\right\}$ and $\left\{\varphi_{j}\right\}$ in $L^{2}[0,1]$. The nature performs step 2 by first selecting a bounded sequence $0<\sigma_{j}<\sigma$ for $j=0,1, \ldots$, sampling $u_{j}$ as i.i.d. $U[-1,1]$, and then setting $\lambda_{j}=u_{j} \sigma_{j}$. Finally, for some scalar $\kappa>0$ it sets

$$
K=\kappa\left(\sum_{j=0}^{\infty} \lambda_{j} \phi_{j} \varphi_{j}\right) .
$$

The operator defined in this way is well-defined over $\mathcal{A}$ and is bounded, but it need not be compact. If compactness is required, we impose $\sum_{j=1}^{\infty} \sigma_{j}^{2}<\infty$ in the construction. If $K \geq 0$ is required, we can impose $\phi_{0}=1, \varphi_{0}=1,\left|\varphi_{j}\right| \leq c$ and $\left|\phi_{j}\right| \leq c$, for all $j$, where $c>1$ is a constant, and $\sum_{j=0}^{\infty} \sigma_{j}<\infty$, and define instead $\lambda_{0}$ as $c \sum_{j=1}^{\infty} \lambda_{j}+\left|u_{0}\right| \sigma_{0}$. If in addition to 
positivity, $\int K(z, t) d t=1$ is required, for example if $K(z, t)=f(t \mid z)$ is a conditional density, then we select $\kappa>0$ so that $\kappa \lambda_{0}=1$. This algorithm for generating $m^{\prime}$ trivially obeys conditions 1 and 2 stated above. Furthermore, $u_{j}$ need not be i.i.d. Take the extreme, opposite example, and set $u_{j}=U[-1,1]$ for all $j$, that is $u_{j}$ 's are perfectly dependent. The resulting algorithm for generating $m^{\prime}$ still trivially obeys conditions 1 and 2 . The latter point - of allowing perfect dependence - is useful for highlighting the differences with the approach and various examples given in Andrews (2011); other than that, our point is the same.

An important example where dependence matters is the case with normal instrumental regression, where the endogenous variable $X$ conditional on the instrument $Z=z$ follows a normal distribution with mean $\rho z$ (and variance normalized to 1 ). Here we let $\mathcal{A}=\mathcal{B}=L^{2}(\mathbb{R}$ ) equipped with standard normal density as a measure. In this case, $m^{\prime}$ is an integral operator

$$
m^{\prime} \delta=\int K(\cdot, t) \delta(t) \frac{1}{\sqrt{2 \pi}} e^{-t^{2} / 2} d t
$$

similarly to what we had above, where $K(t, z)$ has the following well known representation:

$$
K(t, z)=\sum_{j=0}^{\infty} \rho^{j} \phi_{j}(t) \varphi_{j}(z),
$$

where $\left(\phi_{j}\right)_{j=0}^{\infty}$ and $\left(\varphi_{j}\right)_{j=0}^{\infty}$ are the orthonormal (Hermite) polynomials. Hence if the nature draws $\rho$ from an absolutely continuous density on $(-1,1)$, then the full rank condition holds with probability 1. Note that the generalized Fourier coefficients $\left(\rho^{j}\right)_{j=0}^{\infty}$ exhibit perfect dependence here. To see that our randomization algorithm permits this, let the nature draw $\rho$ as specified above and draw $\lambda_{0}$ as an independent from $\rho$ random variable with support $(0, \infty)$ having an absolutely continuous distribution. Then nature sets $\lambda_{j}=\rho^{j} \lambda_{0}, \kappa=1 / \lambda_{0}$, and

$$
K(t, z)=\kappa\left(\sum_{j=0}^{\infty} \lambda_{j} \phi_{j}(z) \varphi_{j}(z)\right) .
$$

\section{B.2 Proof of Lemma 3}

By assumptions there exists a compact, injective operator $\mathcal{K}: \mathcal{A} \mapsto \mathcal{B}$. By Theorem 15.16 in Kress (1999) $\mathcal{K}$ admits a singular value decomposition:

$$
\mathcal{K} \delta=\sum_{j=0}^{N} \mu_{j}\left\langle\phi_{j}, \delta\right\rangle \varphi_{j},
$$


where $\left\{\phi_{j}\right\}$ is an orthonormal subset of $\mathcal{A}$, either finite or countably infinite, with cardinality $N \leq \infty,\left\{\varphi_{j}\right\}$ is an orthonormal subset of $\mathcal{B}$ of equal cardinality, and $\left(\mu_{j}\right)_{j=1}^{\infty}$ is bounded. Since $\|\mathcal{K} \delta\|_{\mathcal{B}}^{2}=\sum_{j=0}^{N} \mu_{j}^{2}\left\langle\phi_{j}, \delta\right\rangle^{2}$, injectivity of $\mathcal{K}$ requires that $\left\{\phi_{j}\right\}$ must be an orthonormal basis in $\mathcal{A}$ and $\mu_{j} \neq 0$ for all $j$. Therefore, step 1 is always feasible by using these $\left\{\phi_{j}\right\}$ and $\left\{\varphi_{j}\right\}$ in the construction. The order of eigenvectors in these sets need not be preserved and could be arbitrary. Step 2 is also feasible by using a product of Lebesgue- dominated measures on a bounded subset of $\mathbb{R}$ to define a measure over $\mathbb{R}^{N}$, or, more generally, using any construction of measure on $\mathbb{R}^{N}$ from finite-dimensional measures obeying Kolmogorov's consistency conditions (e.g. Dudley, 1989) and the additional condition that $\eta\left\{\lambda_{j_{1}} \in A, \lambda_{j_{2}} \in \mathbb{R}, \ldots, \lambda_{j_{k}} \in \mathbb{R}\right\}=0$ if $\operatorname{Leb}(A)=0$, for any finite subset $\left\{j_{1}, \ldots, j_{k}\right\} \subset\{0, \ldots, N\}$. This verifies claim 1 .

To verify claim 2, by Bessel's inequality we have that

$$
\left\|m^{\prime} \delta\right\|_{\mathcal{B}} \geq \sum_{j=0}^{N} \lambda_{j}^{2} \kappa^{2}\left\langle\phi_{j}, \delta\right\rangle^{2}
$$

$m^{\prime}$ is not injective iff $\lambda_{j}^{2} \kappa^{2}=0$ for some $j$. By countable additivity and by $\operatorname{Leb}(\{0\})=0 \Longrightarrow$ $\eta\left(\left\{\lambda_{j}=0\right\}\right)=0$ holding by assumption,

$$
\operatorname{Pr}_{\eta}\left(\exists j \in\{0,1, \ldots, N\}: \lambda_{j}=0\right) \leq \sum_{j=0}^{N} \eta\left(\left\{\lambda_{j}=0\right\}\right)=0 .
$$

The final claim follows from the penultimate display. Q.E.D.

\section{B.3 Proof of Theorem 4}

By Assumption 3, for any $\alpha \neq \alpha_{0}$ and $\alpha \in \mathcal{N}^{\prime \prime \prime}$ with Fourier coefficients $b_{j}$ we have

$$
\left\|m^{\prime}\left(\alpha-\alpha_{0}\right)\right\|_{\mathcal{B}} \geq\left(\sum_{j} \mu_{j}^{2} b_{j}^{2}\right)^{1 / 2}>L\left(\sum_{j} b_{j}^{2}\right)^{r / 2}=L\left\|\alpha-\alpha_{0}\right\|_{\mathcal{A}}^{r},
$$

so the conclusion follows from Theorem 2. Q.E.D.

\section{B.4 Proof of Corollary 5}

Consider $\alpha \in \mathcal{N}^{\prime \prime \prime}$. Then

$$
\sum_{j} \mu_{j}^{-2 /(r-1)} b_{j}^{2}<L^{-2 /(r-1)}
$$


For $b_{j}=\left\langle\alpha-\alpha_{0}, \phi_{j}\right\rangle$ note that $\left\|\alpha-\alpha_{0}\right\|_{\mathcal{A}}=\left(\sum_{j} b_{j}^{2}\right)^{1 / 2}$ by $\phi_{1}, \phi_{2}, \ldots$ being an orthonormal basis. Then

$$
\begin{aligned}
\left(\sum_{j} b_{j}^{2}\right)^{1 / 2} & =\left(\sum_{j} \mu_{j}^{-2 / r} \mu_{j}^{2 / r} b_{j}^{2}\right)^{1 / 2} \leq\left(\sum_{j} \mu_{j}^{-2 /(r-1)} b_{j}^{2}\right)^{(r-1) / 2 r}\left(\sum_{j} \mu_{j}^{2} b_{j}^{2}\right)^{1 / 2 r} \\
& <L^{-1 / r}\left(\sum_{j} \mu_{j}^{2} b_{j}^{2}\right)^{1 / 2 r} \leq L^{-1 / r}\left(\left\|m^{\prime}\left(\alpha-\alpha_{0}\right)\right\|_{\mathcal{B}}\right)^{1 / r}
\end{aligned}
$$

where the first inequality holds by the Hölder inequality, the second by eq. (A.3), and the third by Assumption 3. Raising both sides to the $r^{\text {th }}$ power and multiplying through by $L$ gives

$$
L\left\|\alpha-\alpha_{0}\right\|_{\mathcal{A}}^{r}<\left\|m^{\prime}\left(\alpha-\alpha_{0}\right)\right\|_{\mathcal{B}} .
$$

The conclusion then follows from Theorem 4. Q.E.D.

\section{B.5 Proof of Theorem 6}

Let $F(y \mid X, W)=\operatorname{Pr}(Y \leq y \mid X, W), m(\alpha)=E[1(Y \leq \alpha(X)) \mid W]-\tau$, and

$$
m^{\prime} h=\mathrm{E}\left[f_{Y}\left(\alpha_{0}(X) \mid X, W\right) h(X) \mid W\right]
$$

so that by iterated expectations,

$$
m(\alpha)=\mathrm{E}[F(\alpha(X) \mid X, W) \mid W]-\tau .
$$

Then by a pathwise mean value expansion, and by $f_{Y}(y \mid X, W)$ continuously differentiable

$$
\begin{aligned}
& \left|F(\alpha(X) \mid X, W)-F\left(\alpha_{0}(X) \mid X, W\right)-f_{Y}\left(\alpha_{0}(X) \mid X, W\right)\left(\alpha(X)-\alpha_{0}(X)\right)\right| \\
= & \left|\left[f_{Y}(\bar{\alpha}(X) \mid X, W)-f_{Y}\left(\alpha_{0}(X) \mid X, W\right)\right]\left[\alpha(X)-\alpha_{0}(X)\right]\right| \leq L_{1}\left[\alpha(X)-\alpha_{0}(X)\right]^{2},
\end{aligned}
$$

where $\bar{\alpha}(X)$ is the mean value of a pathwise Taylor expansion that lies between $\alpha(X)$ and $\alpha_{0}(X)$. Then for $L_{1} L_{2}=L$,

$$
\begin{aligned}
\left|m(\alpha)(W)-m\left(\alpha_{0}\right)(W)-m^{\prime}\left(\alpha-\alpha_{0}\right)(W)\right| & \leq L_{1} \mathrm{E}\left[\left\{\alpha(X)-\alpha_{0}(X)\right\}^{2} \mid W\right] \\
& \leq L \mathrm{E}\left[\left\{\alpha(X)-\alpha_{0}(X)\right\}^{2}\right]=L\left\|\alpha-\alpha_{0}\right\|_{\mathcal{A}}^{2} .
\end{aligned}
$$


Therefore,

$$
\left\|m(\alpha)-m\left(\alpha_{0}\right)-m^{\prime}\left(\alpha-\alpha_{0}\right)\right\|_{\mathcal{B}} \leq L\left\|\alpha-\alpha_{0}\right\|_{\mathcal{A}}^{2},
$$

so that Assumption 2 is satisfied with $r=2$ and $\mathcal{N}^{\prime \prime}=\mathcal{A}$. The conclusion then follows from Corollary 5. Q.E.D.

\section{Proofs for Section 4}

\section{C.1 Useful Results on Projections on Linear Subspaces}

Before proving the next Theorem we give two useful intermediate results. Let $\operatorname{Proj}(b \mid \overline{\mathcal{M}})$ denote the orthogonal projection of an element $b$ of a Hilbert space on a closed linear subset $\overline{\mathcal{M}}$ of that space.

Lemma A.1: If a) $\overline{\mathcal{M}}$ is a closed linear subspace of a Hilbert space $\mathcal{H}$; b) $b_{j} \in \mathcal{H}(j=$ $1, \ldots, p)$; c) the $p \times p$ matrix $\Pi$ with $\Pi_{j k}=\left\langle b_{j}-\operatorname{Proj}\left(b_{j} \mid \overline{\mathcal{M}}\right), b_{k}-\operatorname{Proj}\left(b_{k} \mid \overline{\mathcal{M}}\right)\right\rangle$ is nonsingular, then for $b=\left(b_{1}, \ldots, b_{p}\right)^{T}$ there exists $\varepsilon>0$ such that for all $a \in \mathbb{R}^{p}$ and $\zeta \in \overline{\mathcal{M}}$,

$$
\left\|b^{T} a+\zeta\right\| \geq \varepsilon(|a|+\|\zeta\|) .
$$

Proof: Let $\bar{b}_{j}=\operatorname{Proj}\left(b_{j} \mid \overline{\mathcal{M}}\right), \tilde{b}_{j}=b_{j}-\bar{b}_{j}, \bar{b}=\left(\bar{b}_{1}, \ldots, \bar{b}_{p}\right)^{T}$, and $\tilde{b}=\left(\tilde{b}_{1}, \ldots, \tilde{b}_{p}\right)^{T}$. Note that for $\varepsilon_{1}=\sqrt{\lambda_{\min }(\Pi) / 2}$,

$$
\begin{aligned}
\left\|b^{T} a+\zeta\right\| & =\sqrt{\left\|\tilde{b}^{T} a+\zeta+\bar{b}^{T} a\right\|^{2}}=\sqrt{\left\|\tilde{b}^{T} a\right\|^{2}+\left\|\zeta+\bar{b}^{T} a\right\|^{2}} \\
& \geq\left(\left\|\tilde{b}^{T} a\right\|+\left\|\zeta+\bar{b}^{T} a\right\|\right) / \sqrt{2}=\left(\sqrt{a^{T} \Pi a}+\left\|\zeta+\bar{b}^{T} a\right\|\right) / \sqrt{2} \\
& \geq \varepsilon_{1}|a|+\left\|\zeta+\bar{b}^{T} a\right\| / \sqrt{2} .
\end{aligned}
$$

Also note that for any $C^{*} \geq \sqrt{\sum_{j}\left\|\bar{b}_{j}\right\|^{2}}$ it follows by the triangle and Cauchy-Schwartz inequalities that

$$
\left\|\bar{b}^{T} a\right\| \leq \sum_{j}\left\|\bar{b}_{j}\right\|\left|a_{j}\right| \leq C^{*}|a| .
$$

Choose $C^{*}$ big enough that $\varepsilon_{1} / \sqrt{2} C^{*} \leq 1$. Then by the triangle inequality,

$$
\begin{aligned}
\left\|\zeta+\bar{b}^{T} a\right\| / \sqrt{2} & \geq\left(\varepsilon_{1} / \sqrt{2} C^{*}\right)\left\|\zeta+\bar{b}^{T} a\right\| / \sqrt{2}=\varepsilon_{1}\left\|\zeta+\bar{b}^{T} a\right\| / 2 C^{*} \\
& \geq \varepsilon_{1}\left(\|\zeta\|-\left\|\bar{b}^{T} a\right\|\right) / 2 C^{*} \geq \varepsilon_{1}\left(\|\zeta\|-C^{*}|a|\right) / 2 C^{*} \\
& =\left(\varepsilon_{1} / 2 C^{*}\right)\|\zeta\|-\varepsilon_{1}|a| / 2 .
\end{aligned}
$$


Then combining the inequalities, for $\varepsilon=\min \left\{\varepsilon_{1} / 2, \varepsilon_{1} / 2 C^{*}\right\}$,

$$
\begin{aligned}
\left\|b^{T} a+\zeta\right\| & \geq \varepsilon_{1}|a|+\left(\varepsilon_{1} / 2 C^{*}\right)\|\zeta\|-\varepsilon_{1}|a| / 2 \\
& =\left(\varepsilon_{1} / 2\right)|a|+\left(\varepsilon_{1} / 2 C^{*}\right)\|\zeta\| \geq \varepsilon(|a|+\|\zeta\|) . \text { Q.E.D. }
\end{aligned}
$$

Lemma A.2: If Assumption 4 is satisfied then there is an $\varepsilon>0$ such that for all $(\beta, g) \in$ $\mathbb{R}^{p} \times \mathcal{N}_{g}^{\prime}$

$$
\varepsilon\left(\left|\beta-\beta_{0}\right|+\left\|m_{g}^{\prime}\left(g-g_{0}\right)\right\|_{\mathcal{B}}\right) \leq\left\|m^{\prime}\left(\alpha-\alpha_{0}\right)\right\|_{\mathcal{B}}
$$

Proof: Apply Lemma A.1 with $\mathcal{H}$ being the Hilbert space $\mathcal{B}$ described in Section $4, \overline{\mathcal{M}}$ in Lemma A.1 being the closed linear span of $\mathcal{M}=\left\{m_{g}^{\prime}\left(g-g_{0}\right): g \in \mathcal{N}_{g}^{\prime}\right\}, b_{j}=m_{\beta}^{\prime} e_{j}$ for the $j^{\text {th }}$ unit vector $e_{j}$, and $a=\beta-\beta_{0}$. Then for all $(\beta, g) \in \mathbb{R}^{p} \times \mathcal{N}_{g}^{\prime}$ we have

$$
m^{\prime}\left(\alpha-\alpha_{0}\right)=b^{T} a+\zeta, b^{T} a=m_{\beta}^{\prime}\left(\beta-\beta_{0}\right), \zeta=m_{g}^{\prime}\left(g-g_{0}\right) \in \overline{\mathcal{M}}
$$

The conclusion then follows from the conclusion of Lemma A.1. Q.E.D.

\section{C.2 Proof of Theorem 7}

Since Assumption 4 is satisfied the conclusion of Lemma A.2 holds. Let $\varepsilon$ be from the conclusion of Lemma A.2. Also let $\mathcal{N}_{g}=\mathcal{N}_{g}^{\prime} \cap \mathcal{N}_{g}^{\beta}$ for $\mathcal{N}_{g}^{\prime}$ from Assumption 4 and $\mathcal{N}_{g}^{\beta}$ from Assumption 5 . In addition let $B$ be from Assumption 5 with

$$
\sup _{g \in \mathcal{N}_{g}^{\beta}} \mathrm{E}\left[\sup _{\beta \in B}\left|\partial \mathrm{E}[\rho(Y, X, \beta, g) \mid W] / \partial \beta-\partial \mathrm{E}\left[\rho\left(Y, X, \beta_{0}, g_{0}\right) \mid W\right] / \partial \beta\right|^{2}\right]<\varepsilon^{2} .
$$

Then by $m\left(\beta_{0}, g\right)$ linear in $g$ and expanding each element of $m(\beta, g)(W)=\mathrm{E}[\rho(Y, X, \beta, g) \mid W]$ in $\beta$, it follows that for each $(\beta, g) \in B \times \mathcal{N}_{g}$, if $\beta \neq \beta_{0}$,

$$
\begin{aligned}
& \left\|m(\alpha)-m^{\prime}\left(\alpha-\alpha_{0}\right)\right\|_{\mathcal{B}}=\left\|m(\beta, g)-m\left(\beta_{0}, g\right)-m_{\beta}^{\prime}\left(\beta-\beta_{0}\right)\right\|_{\mathcal{B}} \\
= & \left\|\left[\partial m(\tilde{\beta}, g) / \partial \beta-m_{\beta}^{\prime}\right]\left(\beta-\beta_{0}\right)\right\|_{\mathcal{B}} \leq\left\|m_{\beta}^{\prime}(\tilde{\beta}, g)-m_{\beta}^{\prime}\right\|_{\mathcal{B}}\left|\beta-\beta_{0}\right| \\
< & \varepsilon\left|\beta-\beta_{0}\right| \leq \varepsilon\left(\left|\beta-\beta_{0}\right|+\left\|m_{g}^{\prime}\left(g-g_{0}\right)\right\|_{\mathcal{B}}\right) \leq\left\|m^{\prime}\left(\alpha-\alpha_{0}\right)\right\|_{\mathcal{B}},
\end{aligned}
$$

where $\tilde{\beta}$ is a mean value depending on $W$ that actually differs from row to row of

$$
m_{\beta}^{\prime}(\tilde{\beta}, g)=\partial \mathrm{E}[\rho(Y, X, \tilde{\beta}, g) \mid W] / \partial \beta
$$


Thus, $\left\|m(\alpha)-m^{\prime}\left(\alpha-\alpha_{0}\right)\right\|_{\mathcal{B}}<\left\|m^{\prime}\left(\alpha-\alpha_{0}\right)\right\|_{\mathcal{B}}$, implying $m(\alpha) \neq 0$, giving the first conclusion.

To show the second conclusion, consider $(\beta, g) \in \mathcal{N}$. If $\beta \neq \beta_{0}$ then it follows as above that $m(\alpha) \neq 0$. If $\beta=\beta_{0}$ and $g \neq g_{0}$ then by linearity in $g$ we have $\left\|m(\alpha)-m^{\prime}\left(\alpha-\alpha_{0}\right)\right\|_{\mathcal{B}}=0$ while $\left\|m^{\prime}\left(\alpha-\alpha_{0}\right)\right\|_{\mathcal{B}}=\left\|m_{g}^{\prime}\left(g-g_{0}\right)\right\|_{\mathcal{B}}>0$, so $m(\alpha) \neq 0$ follows as in the proof of Theorem 1 . Q.E.D.

\section{C.3 Proof of Theorem 8}

Since Assumption 4 is satisfied the conclusion of Lemma A.2 holds. Let $\varepsilon$ be from the conclusion of Lemma A.2. Define $B$ as in the proof of Theorem 7. By Assumption 2, for $g \in \mathcal{N}_{g}^{\prime \prime}$, $\left\|m\left(\beta_{0}, g\right)-m_{g}^{\prime}\left(g-g_{0}\right)\right\|_{\mathcal{B}} \leq L\left\|g-g_{0}\right\|_{\mathcal{A}}^{r}$. Then similarly to the proof of Theorem 7 for all $\alpha \in \mathcal{N}$ with $\alpha \neq \alpha_{0}$,

$$
\begin{aligned}
& \left\|m(\alpha)-m^{\prime}\left(\alpha-\alpha_{0}\right)\right\|_{\mathcal{B}} \\
\leq & \left\|m(\beta, g)-m\left(\beta_{0}, g\right)-m_{\beta}^{\prime}\left(\beta-\beta_{0}\right)\right\|_{\mathcal{B}}+\left\|m\left(\beta_{0}, g\right)-m_{g}^{\prime}\left(g-g_{0}\right)\right\|_{\mathcal{B}} \\
< & \varepsilon\left|\beta-\beta_{0}\right|+L\left\|g-g_{0}\right\|_{\mathcal{A}}^{r} \leq \varepsilon\left|\beta-\beta_{0}\right|+\varepsilon\left\|m^{\prime}\left(g-g_{0}\right)\right\|_{\mathcal{B}} \\
\leq & \left\|m^{\prime}\left(\alpha-\alpha_{0}\right)\right\|_{\mathcal{B}} .
\end{aligned}
$$

The conclusion follows as in the conclusion of Theorem 1. Q.E.D.

\section{C.4 Proof of Theorem 9}

The proof will proceed by verifying the conditions of Theorem 7. Note that Assumption 4 is satisfied. We now check Assumption 5. Note that for any $\delta>0$ and $g \in \mathcal{N}_{g}^{\delta}, g\left(X_{1}+X_{2}^{T} \beta\right)$ is continuously differentiable in $\beta$ with $\partial g\left(X_{1}+X_{2}^{T} \beta\right) / \partial \beta=g^{\prime}\left(X_{1}+X_{2}^{T} \beta\right) X_{2}$. Also, for $\Delta$ a $p \times 1$ vector and $\bar{B}$ a neighborhood of zero it follows by boundedness of $g_{0}^{\prime}$ and the specification of $\mathcal{N}_{g}^{\delta}$ that for some $C>0$,

$$
\mathrm{E}\left[\sup _{\Delta \in \bar{B}}\left|g^{\prime}\left(X_{1}+X_{2}^{T}(\beta+\Delta)\right) X_{2}\right| \mid W\right] \leq C \mathrm{E}\left[\left|X_{2}\right| \mid W\right]<\infty \text { a.s. }
$$

Therefore, by the dominated convergence theorem $m(\alpha)(W)=\mathrm{E}\left[Y-g\left(X_{1}+X_{2}^{T} \beta\right) \mid W\right]$ is continuously differentiable in $\beta$ a.s. with

$$
\partial m(\alpha)(W) / \partial \beta=-\mathrm{E}\left[g^{\prime}\left(X_{1}+X_{2}^{T} \beta\right) X_{2} \mid W\right] .
$$


Next consider any $\varepsilon>0$ and let $B$ and $\delta$ satisfy

$$
B=\left\{\beta:\left|\beta-\beta_{0}\right|^{2}<\varepsilon^{2} / 4 C_{g}^{2} \mathrm{E}\left[\left|X_{2}\right|^{4}\right]\right\} \text { and } \delta^{2}<\varepsilon^{2} / 4 \mathrm{E}\left[\left|X_{2}\right|^{2}\right]
$$

Then for $g \in \mathcal{N}_{g}^{\delta}$ we have, for $v(X, \beta)=X_{1}+X_{2}^{T} \beta$,

$$
\begin{aligned}
& \mathrm{E}\left[\sup _{\beta \in B}\left|\partial m(\alpha)(W) / \partial \beta-m_{\beta}^{\prime}(W)\right|^{2}\right] \\
= & \mathrm{E}\left[\sup _{\beta \in B}\left|\mathrm{E}\left[\left\{g^{\prime}(v(X, \beta))-g_{0}^{\prime}(V)\right\} X_{2} \mid W\right]\right|^{2}\right] \leq \mathrm{E}\left[\left|X_{2}\right|^{2} \sup _{\beta \in B}\left|g^{\prime}(v(X, \beta))-g_{0}^{\prime}(V)\right|^{2}\right] \\
\leq & 2 \mathrm{E}\left[\left|X_{2}\right|^{2} \sup _{\beta \in B}\left|g^{\prime}(v(X, \beta))-g_{0}^{\prime}(v(X, \beta))\right|^{2}\right]+2 \mathrm{E}\left[\left|X_{2}\right|^{2} \sup _{\beta \in B}\left|g_{0}^{\prime}(v(X, \beta))-g_{0}^{\prime}(V)\right|^{2}\right] \\
\leq & 2 \delta^{2} \mathrm{E}\left[\left|X_{2}\right|^{2}\right]+2 C_{g}^{2} \mathrm{E}\left[\left|X_{2}\right|^{4}\right] \sup _{\beta \in B}\left|\beta-\beta_{0}\right|^{2}<\varepsilon^{2} .
\end{aligned}
$$

Thus Assumption 5 is satisfied so the first conclusion follows by the first conclusion of Theorem 7. Also, $m_{g}^{\prime}\left(g-g_{0}\right)=E\left[g(V)-g_{0}(V) \mid W\right]$ the rank condition for $m_{g}^{\prime}$ follows by the last bounded completeness on $\mathcal{N}_{g}^{\prime}$, so that the final conclusion follows by the final conclusion of Theorem 7 . Q.E.D.

\section{C.5 Proof of Theorem 10}

Suppose first that the conditional distribution of $W$ given $V$ is complete. Note that by the projection definition, for all $h(V)$ with finite mean-square we have

$$
0=\mathrm{E}\left[\left\{-\mathrm{E}\left[g_{0}^{\prime}(V) X_{2 j} \mid W\right]-\zeta_{j}^{*}(W)\right\} \mathrm{E}[h(V) \mid W]\right]=\mathrm{E}\left[\left\{-\mathrm{E}\left[g_{0}^{\prime}(V) X_{2 j} \mid W\right]-\zeta_{j}^{*}(W)\right\} h(V)\right] .
$$

Therefore,

$$
\mathrm{E}\left[-\mathrm{E}\left[g_{0}^{\prime}(V) X_{2 j} \mid W\right]-\zeta_{j}^{*}(W) \mid V\right]=0 .
$$

Completeness of the conditional distribution of $W$ given $V$ then implies that $-\mathrm{E}\left[g_{0}^{\prime}(V) X_{2 j} \mid W\right]-$ $\zeta_{j}^{*}(W)=0$, and hence $\Pi_{j j}=0$. Since this is true for each $j$ we have $\Pi=0, \Pi$ is singular.

Next, consider the second hypothesis and $\lambda \neq 0$. Let $\zeta_{\lambda}^{*}(W)$ denote the projection of $-\mathrm{E}\left[g_{0}^{\prime}(V) \lambda^{T} X_{2} \mid W\right]$ on $\overline{\mathcal{M}}$. Since $\mathrm{E}[h(V) \mid W]=\mathrm{E}[h(V) \mid T(W)]$ it follows that $\zeta_{\lambda}^{*}(W)$ is measurable with respect to (i.e. is a function of) $T(W)$. Since $\mathrm{E}\left[g_{0}^{\prime}(V) \lambda^{T} X_{2} \mid W\right]$ is not measurable with respect to $T(W)$, we have $-\mathrm{E}\left[g_{0}^{\prime}(V) \lambda^{T} X_{2} \mid W\right]-\zeta_{\lambda}^{*}(W) \neq 0$, so that

$$
\lambda^{T} \Pi \lambda=\mathrm{E}\left[\left\{-\mathrm{E}\left[g_{0}^{\prime}(V) \lambda^{T} X_{2} \mid W\right]-\zeta_{\lambda}^{*}(W)\right\}^{2}\right]>0 .
$$


Since this is true for all $\lambda \neq 0$, it follows that $\Pi$ is positive definite, and hence nonsingular. Q.E.D.

\section{Proofs for Section 5}

\section{D.1 Proof of Theorem 11}

The proof will proceed by verifying the conditions of Theorem 7 for $\rho(Y, \beta, g)$ from eq. (5.7). We first check first part of Assumption 4. Note that the mapping $m^{\prime}: \mathbb{R}^{2} \times \mathcal{G} \longrightarrow \mathcal{B}$ is given by $m^{\prime}\left(\alpha-\alpha_{0}\right)=m_{\beta}^{\prime}\left(\beta-\beta_{0}\right)+m_{g}^{\prime}\left(g-g_{0}\right)$, where

$$
\begin{aligned}
& m_{\beta}^{\prime}\left(\beta-\beta_{0}\right)=\mathrm{E}\left[A_{t} g_{0}\left(c_{t+1}\right) X_{t}^{T} \mid W_{t}\right]\left(\beta-\beta_{0}\right) \text { and } \\
& m_{g}^{\prime}\left(g-g_{0}\right)=\mathrm{E}\left[A_{t}\left\{g\left(c_{t+1}\right)-g_{0}\left(c_{t+1}\right)\right\} \mid W_{t}\right]-\left\{g\left(c_{t}\right)-g_{0}\left(c_{t}\right)\right\} e
\end{aligned}
$$

Therefore the mapping $m^{\prime}$ is obviously linear. Since $\mathrm{E}\left[D_{t}^{2} \mid W_{t}\right]$ and $\mathrm{E}\left[D_{t} \mid W_{t}\right]$ exist with probability one by $\mathrm{E}\left[D_{t}^{2}\right]<\infty$ and that $\left|A_{t}\right|^{2} \leq C D_{t}^{2}$. Then by the Cauchy-Schwartz inequality, for any $h \in \mathcal{G}$ we have by $D_{t} \geq 1, \mathrm{E}\left[D_{t}^{2} \mid W_{t}\right] \geq 1$.

$$
\begin{aligned}
& \left\|\mathrm{E}\left[A_{t} h\left(c_{t+1}\right) \mid W_{t}\right]-h\left(c_{t}\right) e\right\|_{\mathcal{B}}^{2} \leq C \mathrm{E}\left[\mathrm{E}\left[A_{t}^{T} h\left(c_{t+1}\right) \mid W_{t}\right] \mathrm{E}\left[A_{t} h\left(c_{t+1}\right) \mid W_{t}\right]+h\left(c_{t}\right)^{2}\right] \\
\leq & C \mathrm{E}\left[\mathrm{E}\left[D_{t}^{2} \mid W_{t}\right] \mathrm{E}\left[h\left(c_{t+1}\right)^{2} \mid W_{t}\right]+C \mathrm{E}\left[\mathrm{E}\left[D_{t-1}^{2} \mid W_{t-1}\right] h\left(c_{t}\right)^{2}\right] \leq C\|h\|_{\mathcal{G}}^{2} .\right.
\end{aligned}
$$

Thus $m_{g}^{\prime}: \mathcal{G} \longrightarrow \mathcal{B}$ is bounded. Also, noting that $\left|m_{\beta}^{\prime}\right| \leq \mathrm{E}\left[D_{t} g_{0}\left(c_{t+1}\right) \mid W_{t}\right]$, the CauchySchwartz inequality gives

$$
\mathrm{E}\left[\left|m_{\beta}^{\prime}(W)\right|^{2}\right] \leq \mathrm{E}\left[\mathrm{E}\left[D_{t}^{2} \mid W_{t}\right] \mathrm{E}\left[g_{0}\left(c_{t+1}\right)^{2} \mid W_{t}\right]\right] \leq\left\|g_{0}\right\|_{\mathcal{G}}^{2}<\infty
$$

and hence $m_{\beta}^{\prime}: \mathbb{R}^{2} \longrightarrow \mathcal{B}$ is bounded. Therefore the first part of Assumption 4 is satisfied with $\mathcal{N}_{g}^{\prime}=\mathcal{G}$.

Turning now to Assumption 5. Let $H_{t}(\beta, g)=\delta R_{t+1} c_{t+1}^{-\gamma} g\left(c_{t+1}\right)$ and $B=\left[\delta_{0}-\Delta, \delta_{0}+\Delta\right] \times$ $\left[\gamma_{0}-\Delta, \gamma_{0}+\Delta\right]$. Note that $H_{t}(\beta, g)$ is twice continuously differentiable in $\beta$ and by construction of $D_{t}$ that

$$
\sup _{\beta \in B}\left|\frac{\partial H_{t}(\beta, g)}{\partial \beta}\right| \leq D_{t} g\left(c_{t+1}\right), \sup _{\beta \in B}\left|\frac{\partial^{2} H_{t}(\beta, g)}{\partial \beta_{j} \partial \beta}\right| \leq D_{t} g\left(c_{t+1}\right), \quad(j=1,2) .
$$


Therefore by standard results $\mathrm{E}\left[\rho\left(Y_{t}, \beta, g\right) \mid W_{t}\right]=\mathrm{E}\left[H_{t}(\beta, g) \mid W_{t}\right]-g\left(c_{t}\right)$ is twice continuously differentiable in $\beta$ on $B, \partial \mathrm{E}\left[\rho\left(Y_{t}, \beta, g\right) \mid W_{t}\right] / \partial \beta=\mathrm{E}\left[\partial H_{t}(\beta, g) / \partial \beta \mid W_{t}\right]$. We also have

$$
\begin{aligned}
\left|\mathrm{E}\left[\partial H_{t}(\beta, g) / \partial \beta-\partial H_{t}\left(\beta, g_{0}\right) / \partial \beta \mid W_{t}\right]\right|^{2} & \leq \mathrm{E}\left[D_{t}^{2} \mid W_{t}\right] \mathrm{E}\left[\left|g\left(c_{t+1}\right)-g_{0}\left(c_{t+1}\right)\right|^{2} \mid W_{t}\right] \\
\left|\mathrm{E}\left[\partial H_{t}\left(\beta, g_{0}\right) / \partial \beta-\partial H_{t}\left(\beta_{0}, g_{0}\right) / \partial \beta \mid W_{t}\right]\right|^{2} & \leq \mathrm{E}\left[D_{t}^{2} \mid W_{t}\right] \mathrm{E}\left[g_{0}\left(c_{t+1}\right)^{2} \mid W_{t}\right]\left|\beta-\beta_{0}\right|^{2} .
\end{aligned}
$$

Therefore we have

$$
\begin{aligned}
& \left|\frac{\partial \mathrm{E}[\rho(Y, \beta, g) \mid W]}{\partial \beta}-\frac{\partial \mathrm{E}\left[\rho\left(Y, \beta_{0}, g_{0}\right) \mid W\right]}{\partial \beta}\right|^{2} \\
= & \left|\mathrm{E}\left[\partial H_{t}(\beta, g) / \partial \beta-\partial H_{t}\left(\beta_{0}, g_{0}\right) / \partial \beta \mid W_{t}\right]\right|^{2} \\
\leq & 2 \mathrm{E}\left[D_{t}^{2} \mid W_{t}\right]\left\{\mathrm{E}\left[\left|g\left(c_{t+1}\right)-g_{0}\left(c_{t+1}\right)\right|^{2} \mid W_{t}\right]+\mathrm{E}\left[g_{0}\left(c_{t+1}\right)^{2} \mid W_{t}\right]\left|\beta-\beta_{0}\right|^{2}\right\} .
\end{aligned}
$$

Note that by iterated expectations,

$$
\begin{aligned}
\mathrm{E}\left[\mathrm{E}\left[D_{t}^{2} \mid W_{t}\right] \mathrm{E}\left[\left|g\left(c_{t+1}\right)-g_{0}\left(c_{t+1}\right)\right|^{2} \mid W_{t}\right]\right] & =\left\|g-g_{0}\right\|_{\mathcal{G}}^{2}, \\
\mathrm{E}\left[\mathrm{E}\left[D_{t}^{2} \mid W_{t}\right] \mathrm{E}\left[\left.g_{0}\left(c_{t+1}\right)\right|^{2} \mid W_{t}\right]\right] & =\left\|g_{0}\right\|_{\mathcal{G}}^{2} .
\end{aligned}
$$

Consider any $\varepsilon>0$. Let

$$
\mathcal{N}_{g}^{\beta}=\left\{g:\left\|g-g_{0}\right\|_{\mathcal{G}} \leq \varepsilon / 2\right\} \text { and } \tilde{B}=B \cap\left\{\beta:\left|\beta-\beta_{0}\right|<\varepsilon /\left(2\left\|g_{0}\right\|_{\mathcal{G}}\right)\right.
$$

Then for $g \in \mathcal{N}_{g}^{\beta}$ we have

$$
\mathrm{E}\left[\sup _{\beta \in \tilde{B}}\left|\partial m(\alpha)(W) / \partial \beta-m_{\beta}^{\prime}(W)\right|^{2}\right] \leq 2\left\|g-g_{0}\right\|_{\mathcal{G}}^{2}+2\left\|g_{0}\right\|_{\mathcal{G}}^{2} \sup _{\beta \in \tilde{B}}\left|\beta-\beta_{0}\right|^{2}<\varepsilon^{2} .
$$

Therefore Assumption 5 holds with $B$ there equal to $\tilde{B}$ here. The conclusion then follows from Theorem 7. Q.E.D.

\section{D.2 Proof of Theorem 12}

Let $\bar{a}\left(c_{t+1}, \tilde{W}_{t}\right)=\mathrm{E}\left[A_{t j} \mid c_{t+1}, \tilde{W}_{t}\right]$ and $\bar{d}\left(c_{t+1}\right)=\mathrm{E}\left[\mathrm{E}\left[D_{t}^{2} \mid \tilde{W}_{t}\right] \mid c_{t+1}\right]$. Let $\tilde{\mathcal{B}}=\left\{b\left(\tilde{W}_{t}\right): \mathrm{E}\left[b\left(\tilde{W}_{t}\right)^{2}\right]<\right.$ $\infty\}$ and the operator $L: \mathcal{G} \longrightarrow \tilde{\mathcal{B}}$ be given by

$$
\begin{aligned}
L g & =\mathrm{E}\left[A_{t j} g\left(c_{t+1}\right) \mid \tilde{W}_{t}\right]=\int \bar{a}\left(c, \tilde{W}_{t}\right) g(c) \frac{f_{c, \tilde{W}}\left(c, \tilde{W}_{t}\right)}{f_{\tilde{W}}\left(\tilde{W}_{t}\right)} d c \\
& =\int g(c) K\left(c, \tilde{W}_{t}\right) f_{c}(c) \bar{d}(c) d c, \quad K\left(c, \tilde{W}_{t}\right)=\frac{\bar{a}\left(c, \tilde{W}_{t}\right) f_{c, \tilde{W}}\left(c, \tilde{W}_{t}\right)}{f_{\tilde{W}}\left(\tilde{W}_{t}\right) f_{c}(c) \bar{d}(c)} .
\end{aligned}
$$


Note that $\bar{d}(c) \geq 1$ by $D_{t}^{2} \geq 1$. Therefore,

$$
\begin{aligned}
\int K(c, w)^{2} \bar{d}(c) f_{c}(c) f_{\tilde{W}}(w) d c d w & =\int \frac{\bar{a}(c, w)^{2} f_{c, \tilde{W}}(c, w)}{f_{\tilde{W}}(w) f_{c}(c) \bar{d}(c)} f_{c, \tilde{W}}(c, w) d c d w \\
& \leq \int \frac{\bar{a}(c, w)^{2} f_{c, \tilde{W}}(c, w)}{f_{\tilde{W}}(w) f_{c}(c)} f_{c, \tilde{W}}(c, w) d c d w \\
& =\mathrm{E}\left[\mathrm{E}\left[A_{t j} \mid c_{t+1}, \tilde{W}_{t}\right]^{2} \frac{f_{c, \tilde{W}}\left(c_{t+1}, \tilde{W}_{t}\right)}{f_{c}\left(c_{t+1}\right) f_{\tilde{W}}\left(\tilde{W}_{t}\right)}\right] \\
& \leq \mathrm{E}\left[A_{t j}^{2} f_{c}\left(c_{t+1}\right)^{-1} f_{\tilde{W}}\left(\tilde{W}_{t}\right)^{-1} f_{c, \tilde{W}}\left(c_{t+1}, \tilde{W}_{t}\right)\right]<\infty
\end{aligned}
$$

It therefore follows by standard results that $L$ is Hilbert-Schmidt and thus compact. Furthermore, it follows exactly as in the proof of Theorem 3.2 of Kress (1999), that

$$
\tilde{\mathcal{M}}=\left\{\mathrm{E}\left[A_{t j} g\left(c_{t+1}\right) \mid \tilde{W}_{t}\right]-g\left(c_{t}\right): g \in \mathcal{G}\right\}
$$

is closed.

Next let $b=\left(b_{1}, b_{2}\right)^{T}$ be a constant vector and $\Delta(c)=b_{1} / \delta_{0}-b_{2} \ln (c)$. Suppose $b^{T} \Pi b=0$. Then by the definition of $\Pi$ there is $g_{k} \in \mathcal{G}$ such that

$$
\mathrm{E}\left[A_{t} g_{k}\left(c_{t+1}\right) \mid \tilde{W}_{t}\right]-g_{k}\left(c_{t}\right) e \longrightarrow \mathrm{E}\left[A_{t} g_{0}\left(c_{t+1}\right) \Delta\left(c_{t+1}\right) \mid \tilde{W}_{t}\right]
$$

in mean square as $k \longrightarrow \infty$. It follows that for any $j$,

$$
\mathrm{E}\left[A_{t j} g_{k}\left(c_{t+1}\right) \mid \tilde{W}_{t}\right]-g_{k}\left(c_{t}\right) \longrightarrow \mathrm{E}\left[A_{t j} g_{0}\left(c_{t+1}\right) \Delta\left(c_{t+1}\right) \mid \tilde{W}_{t}\right]
$$

in mean square. By $\tilde{\mathcal{M}}$ a closed set there exists $g^{*}(c)$ such that

$$
\mathrm{E}\left[A_{t j} g_{0}\left(c_{t+1}\right) \Delta\left(c_{t+1}\right) \mid \tilde{W}_{t}\right]=\mathrm{E}\left[A_{t j} g^{*}\left(c_{t+1}\right) \mid \tilde{W}_{t}\right]-g^{*}\left(c_{t}\right)
$$

If $g^{*}\left(c_{t+1}\right)=0$ then $\mathrm{E}\left[A_{t j} g_{0}\left(c_{t+1}\right) \Delta\left(c_{t+1}\right) \mid \tilde{W}_{t}\right]=0$ and by completeness of $\mathrm{E}\left[A_{t j} h\left(c_{t+1}, c_{t}\right) \mid \tilde{W}_{t}\right]$ it follows that $g_{0}\left(c_{t+1}\right) \Delta\left(c_{t+1}\right)=0$. Then by $\operatorname{Pr}\left(g_{0}\left(c_{t+1}\right) \neq 0\right)=1$, we have $\Delta\left(c_{t+1}\right)=0$.

Next, suppose $\operatorname{Pr}\left(g^{*}\left(c_{t}\right) \neq 0\right)>0$. Then $\operatorname{Pr}\left(\min \left\{\left|g^{*}\left(c_{t}\right)\right|, g_{0}\left(c_{t}\right)\right\}>0\right)>0$, so for small enough $\varepsilon>0$ and $\mathcal{C}=\left\{c_{t}: \min \left\{\left|g^{*}\left(c_{t}\right)\right|, g_{0}\left(c_{t}\right)\right\} \geq \varepsilon\right\}$ we have $\operatorname{Pr}(\mathcal{C})>0$. Let $\left.1_{t}^{\varepsilon}=1\left(c_{t} \in \mathcal{C}\right)\right)$. Then multiplying through eq. (A.5) by $1_{t}^{\varepsilon} / g^{*}\left(c_{t}\right)$ and subtracting the conditional expectation on the right-hand side gives

$$
\mathrm{E}\left[A_{t j} 1_{t}^{\varepsilon} \frac{g_{0}\left(c_{t+1}\right) \Delta\left(c_{t+1}\right)-g^{*}\left(c_{t+1}\right)}{-g^{*}\left(c_{t}\right)} \mid \tilde{W}_{t}\right]=1_{t}^{\varepsilon}
$$


By eq. (5.5) we also have

$$
\mathrm{E}\left[A_{t j} 1_{t}^{\varepsilon}\left\{\frac{g_{0}\left(c_{t+1}\right)}{g_{0}\left(c_{t}\right)}\right\} \mid \tilde{W}_{t}\right]=1_{t}^{\varepsilon}
$$

By the completeness condition in part a) it then follows that

$$
1_{t}^{\varepsilon} \frac{g_{0}\left(c_{t+1}\right) \Delta\left(c_{t+1}\right)-g^{*}\left(c_{t+1}\right)}{-g^{*}\left(c_{t}\right)}=1_{t}^{\varepsilon} \frac{g_{0}\left(c_{t+1}\right)}{g_{0}\left(c_{t}\right)} .
$$

Multiplying, dividing, and subtracting gives

$$
1_{t}^{\varepsilon}\left[\frac{g_{0}\left(c_{t+1}\right) \Delta\left(c_{t+1}\right)-g^{*}\left(c_{t+1}\right)}{-g_{0}\left(c_{t+1}\right)}-\frac{g^{*}\left(c_{t}\right)}{g_{0}\left(c_{t}\right)}\right]=0 .
$$

Then by the additive separability condition in part a) of the conditions it follows that $g_{0}\left(c_{t+1}\right) \Delta\left(c_{t+1}\right)-$ $g^{*}\left(c_{t+1}\right)=C g_{0}\left(c_{t+1}\right)$ for some nonzero constant $C$. Then by eq. (A.5) and the second kind equation for $g_{0}$ we have

$$
g^{*}\left(c_{t}\right)=-\mathrm{E}\left[A_{t j}\left\{g_{0}\left(c_{t+1}\right) \Delta\left(c_{t+1}\right)-g^{*}\left(c_{t+1}\right)\right\} \mid \tilde{W}_{t}\right]=-C \mathrm{E}\left[A_{t j} g_{0}\left(c_{t+1}\right) \mid \tilde{W}_{t}\right]=-C g_{0}\left(c_{t}\right) .
$$

Then by eq. (A.5),

$\mathrm{E}\left[A_{t j} g_{0}\left(c_{t+1}\right) \Delta\left(c_{t+1}\right) \mid \tilde{W}_{t}\right]=\mathrm{E}\left[A_{t j} g^{*}\left(c_{t+1}\right) \mid \tilde{W}_{t}\right]-g^{*}\left(c_{t}\right)=-C\left\{\mathrm{E}\left[A_{t j} g_{0}\left(c_{t+1}\right) \mid \tilde{W}_{t}\right]-g_{0}\left(c_{t}\right)\right\}=0$

By the completeness condition in part a) of the conditions it follows that $g_{0}\left(c_{t+1}\right) \Delta\left(c_{t+1}\right)=0$ so $\Delta\left(c_{t+1}\right)=0$ follows by $\operatorname{Pr}\left(g_{0}\left(c_{t}\right)=0\right)=0$. Therefore, we find that $b^{T} \Pi b=0$ implies $\Delta\left(c_{t+1}\right)=0$. But we know that for $b \neq 0$ it is the case that $\Delta\left(c_{t+1}\right) \neq 0$. Therefore, $b \neq 0$ implies $b^{T} \Pi b>0$, i.e. $\Pi$ is nonsingular.

Next, under condition (b) of Theorem 12 , if $\mathrm{E}\left[A_{t j} g\left(c_{t+1}\right) \mid \tilde{W}_{t}\right]=g\left(c_{t}\right)$ for $g \in \mathcal{G}_{\bar{c}}$ it follows that for $\bar{c}$ as given there,

$$
\mathrm{E}\left[A_{t j} \frac{g\left(c_{t+1}\right)}{g(\bar{c})} \mid w\left(Z_{t}\right), c_{t}=\bar{c}\right]=1=\mathrm{E}\left[A_{t j} \frac{g_{0}\left(c_{t+1}\right)}{g_{0}(\bar{c})} \mid w\left(Z_{t}\right), c_{t}=\bar{c}\right]
$$

Then by the completeness condition in part (b) of the hypotheses, it follows that $g\left(c_{t+1}\right) / g(\bar{c})=$ $g_{0}\left(c_{t+1}\right) / g_{0}(\bar{c})$, i.e.

$$
g\left(c_{t+1}\right)=g_{0}\left(c_{t+1}\right) g(\bar{c}) / g_{0}(\bar{c})
$$

so $g$ is equal to $g_{0}$ up to scale. This also implies that $g_{0}$ is the unique solution to $\mathrm{E}\left[A_{t} g\left(c_{t+1}\right) \mid W_{t}\right]=$ $g\left(c_{t}\right)$ up to scale. Q.E.D. 


\section{D.3 Completeness and Global Identification in the CCAPM}

Theorem A.3. Consider model (5.5). If $\left(R_{t, j}, c_{t}\right)$ is strictly stationary, $c_{t}$ is continuously dis-

tributed with support $[0, \infty), g_{0}(c) \geq 0$ is bounded and bounded away from zero, $\mathrm{E}\left[\left|R_{t+1, j} c_{t}^{-\gamma_{0}}\right|\right]<$ $\infty$, and there is $\bar{c}$ such that $\mathrm{E}\left[R_{t+1, j} h\left(c_{t+1}\right) \mid w\left(Z_{t}\right), \bar{c}\right]=0$ and $\mathrm{E}\left[\left|R_{t+1, j} h\left(c_{t+1}\right)\right|\right]<\infty$ implies $h\left(c_{t+1}\right)=0$ then $\left(\delta_{0}, \gamma_{0}, g_{0}\right)$ is identified ( $g_{0}$ up to scale) among all $(\delta, \gamma, g)$ with $g(c) \geq 0, g(c)$ bounded and bounded away from zero, and $\mathrm{E}\left[\left|R_{t+1, j} c_{t}^{-\gamma}\right|\right]<\infty$.

Proof: Consider any two solutions $\left(\beta_{0}, g_{0}\right)$ and $\left(\beta_{1}, g_{1}\right)$ to equation (5.5) satisfying the conditions of Theorem A.3. Then by iterated expectations,

$$
\mathrm{E}\left[R_{t+1, j} \delta_{0} c_{t+1}^{-\gamma_{0}} \frac{g_{0}\left(c_{t+1}\right)}{g_{0}(\bar{c})} \mid w\left(Z_{t}\right), \bar{c}\right]=1=\mathrm{E}\left[R_{t+1, j} \delta_{1} c_{t+1}^{-\gamma_{1}} \frac{g_{1}\left(c_{t+1}\right)}{g_{1}(\bar{c})} \mid w\left(Z_{t}\right), \bar{c}\right] .
$$

By completeness with $h\left(c_{t+1}\right)=\delta_{0} c_{t+1}^{-\gamma_{0}} g_{0}\left(c_{t+1}\right) / g_{0}(\bar{c})-\delta_{1} c_{t+1}^{-\gamma_{1}} g_{1}\left(c_{t+1}\right) / g_{1}(\bar{c})$ it follows by multiplying and dividing that

$$
c_{t+1}^{\gamma_{1}-\gamma_{0}}=\frac{g_{1}\left(c_{t+1}\right)}{g_{0}\left(c_{t+1}\right)}\left[\frac{\delta_{1} g_{0}(\bar{c})}{\delta_{0} g_{1}(\bar{c})}\right] .
$$

Since the object on the right is bounded and bounded away from zero and the support of $c_{t+1}$ is $I=[0, \infty)$ it follows that $\gamma_{0}=\gamma_{1}$. Then we have

$$
g_{0}\left(c_{t+1}\right)=g_{1}\left(c_{t+1}\right)\left[\frac{\delta_{1} g_{0}(\bar{c})}{\delta_{0} g_{1}(\bar{c})}\right] \text { a.e. in } I^{2}
$$

so that there is a constant $D>0$ such that $g_{0}\left(c_{t+1}\right)=D g_{1}\left(c_{t+1}\right)$ a.e. in $I$. We can also assume that $g_{0}(\bar{c})=D g_{1}(\bar{c})$ since $c_{t}$ is continuously distributed. Substituting then gives $D=\left(\delta_{1} / \delta_{0}\right) D$, implying $\delta_{1}=\delta_{0}$. Q.E.D.

Previously Chen and Ludvigson (2009) show global identification of $\left(\delta_{0}, \gamma_{0}, g_{0}\right)$ under different conditions. In their results $\mathrm{E}\left[R_{t+1, j} h\left(c_{t+1}, c_{t}\right) \mid w\left(Z_{t}\right), c_{t}\right]$ is assumed to be complete, which is similar to condition (a) in Theorem 12 and is stronger than completeness at $c_{t}=\bar{c}$, but $g(c)$ is not assumed to be bounded or bounded away from zero on $[0, \infty)$.

\section{D.4 A Useful Result on Uniqueness and Existence of Positive Eigenfunctions}

The following result and its proof in part rely on the fundamental results of Krein and Rutman (1950), specifically their Theorem 6.1 and example $\beta^{\prime}$. Krein and Rutman (1950) is one of many 
extensions of the Perron-Frobenius theory of positive matrices to the case of operators leaving invariant a cone in a Banach space.

Let $I$ be a Borel subset of $\mathbb{R}^{m}$ and $\mu$ be a $\sigma$-finite measure with support $I$. Consider the space $L^{2}(\mu)$, equipped with the standard norm $\|\cdot\|$. We consider the following conditions on the kernel $K$ :

1. $K(s, t)$ is a non-negative, measurable kernel such that $\iint K^{2}(s, t) d \mu(t) d \mu(s)<\infty$.

2. $K(s, t)=0$ on a set of points $(t, s)$ of measure zero under $\mu \times \mu$.

Consider an integral operator $L$ from $L^{2}(\mu)$ to $L^{2}(\mu)$ defined by:

$$
L \varphi:=\int K(\cdot, t) \varphi(t) d \mu(t)
$$

and its adjoint operator

$$
L^{*} \psi:=\int K(t, \cdot) \psi(t) d \mu(t)
$$

It is known that these operators are compact under condition 1. The lemma given below shows that under these assumptions we have existence and global uniqueness of the positive eigenpair $(\rho, \varphi)$ such that $L \varphi=\rho \varphi$, in the sense that is stated below. This lemma extends example $\beta^{\prime}$ outlined in Krein and Rutman (1950) that looked at the complex Hilbert space $L^{2}[a, b]$, $0<a<b<\infty$, an extension which we were not able to track easily in the literature, so we simply derived it; we also provided an additional step (3), not given in the outline, to fully verify uniqueness. Note that we removed the complex analysis based arguments, since they are not needed here.

LEMmA A.4. Under conditions 1 and 2, there exists a unique eigenpair $(\rho, \varphi)$, consisting of an eigenvalue $\rho$ and eigenfunction $\varphi$ such that $L \varphi=\rho \varphi$ and $\rho>0,\|\varphi\|=1, \varphi \geq 0$; moreover, $\varphi>0 \mu$-a.e.

Proof. The proof is divided in five steps.

(1) Let $C^{o}$ be the cone of nonnegative functions in $A=L^{2}(\mu)$. In the proof we shall use the following result on the existence of non-negative eigenpair from Krein and Rutman (1950, Theorem 6.1). 
Consider a cone $C^{o}$ in a Banach space $A$ such that the closure of the linear hull of $C^{o}$ is $A$. Consider a linear, compact operator $L: A \mapsto A$ such that $L C^{o} \subset C^{o}$, and that has one point of spectrum different from zero. Then it has a positive eigenvalue $\rho$, not less in modulus than every other eigenvalues, and to this eigenvalue there corresponds at least one eigenvector $\varphi \in C^{o}$ of the operator $L(L \varphi=\rho \varphi)$ and at least one eigenvector $\psi \neq 0$ of the dual operator $L^{*}\left(L^{*} \psi=\rho \psi\right)$.

The theorem requires that the closure of the linear hull of the cone is $A$. This is true in our case for $A=L^{2}(\mu)$ and the cone $C^{o}$ of the non-negative functions in $A$, since $C^{o}-C^{o}$ is dense in $A$. Moreover, since

$$
\sigma_{2}=\int K(s, t) K(t, s) d \mu(s) d \mu(t)>0
$$

which is equal to sum of squared eigenvalues of $L$, the spectrum of $L$ must have at least one point different from zero. Therefore, application of the theorem quoted above implies that there exists $\rho>0$ and $\varphi$ and $\psi$ s.t. $\mu$-a.e.

$$
\begin{aligned}
& \varphi(s)=\rho^{-1} \int K(s, t) \varphi(t) d \mu(t), \quad \varphi \geq 0,\|\varphi\|=1, \rho>0 \\
& \psi(s)=\rho^{-1} \int K(t, s) \psi(t) d \mu(t), \quad\|\psi\|=1
\end{aligned}
$$

(2) We would like to prove that any eigenvalue $\rho>0$ associated to a nonnegative eigenfunction $\varphi \geq 0$ must be a simple eigenvalue, i.e. $\varphi$ is the only eigenfunction in $L^{2}(\mu)$ associated with $\rho$. For this purpose we shall use the following standard fact on linear compact operators, e.g. stated in Krein and Rutman (1950) and specialized to our context: An eigenvalue $\rho$ of $L$ is simple if and only if the equations $L \varphi=\rho \varphi$ and $L^{*} \psi=\rho \psi$ have no solutions orthogonal to each other, i.e. satisfying $\varphi \neq 0, \psi \neq 0, \int \psi(s) \varphi(s) d \mu(s)=0$. So for this purpose we will show in steps (4) and (5) below that $\psi$ is of constant sign $\mu$-a.e. and $\varphi$ and $\psi$ only vanish on a set of measure 0 under $\mu$. Since $\varphi \geq 0$, this implies

$$
\int \psi(s) \varphi(s) d \mu(s) \neq 0
$$

and we conclude from the quoted fact that $\rho$ is a simple eigenvalue. 
(3) To assert the uniqueness of the nonnegative eigenpair $(\rho, \varphi)$ (meaning that $L \varphi=\rho \varphi$, $\rho>0, \varphi \geq 0,\|\varphi\|=1$ ), suppose to the contrary that there is another nonnegative eigenpair $(r, \zeta)$. Then $r$ is also an eigenvalue of $L^{*}$ by the Fredholm theorem (Kress, 1999, Theorem 4.14), which implies by definition of the eigenvalue that there exists a dual eigenfunction $\eta \neq 0$ such that $L^{*} \eta=r \eta$ and $\|\eta\|=1$.

By step (4) below we must have $\zeta>0, \varphi>0 \mu$-a.e. Hence by step (5) the dual eigenfunctions $\eta$ and $\psi$ are non-vanishing and of constant sign $\mu$-a.e., which implies $\int \eta(s) \varphi(s) d \mu(s) \neq 0$. Therefore, $r=\rho$ follows from the equality:

$$
r \int \eta(s) \varphi(s) d \mu(s)=\iint K(t, s) \eta(t) d \mu(t) \varphi(s) d \mu(s)=\rho \int \eta(t) \varphi(t) d \mu(t) .
$$

(4) Let us prove that any eigenfunction $\varphi \geq 0$ of $L$ associated with an eigenvalue $\rho>0$ must be $\mu$-a.e. positive. Let $S$ denote the set of zeros of $\varphi$. Evidently, $\mu(S)<\mu(I)$. If $s \in S$, then

$$
\int K(s, t) \varphi(t) d \mu(t)=0
$$

Therefore $K(s, t)$ vanishes almost everywhere on $(s, t) \in S \times(I \backslash S)$. However the set of zeroes of $K(s, t)$ is of measure zero under $\mu \times \mu$, so $\mu(S) \times \mu(I \backslash S)=0$, implying $\mu(S)=0$.

(5) Here we show that any eigen-triple $(\rho, \varphi, \psi)$ solving (A.7) and (A.6) obeys:

$$
\operatorname{sign}(\psi(s))=1 \mu \text {-a.e. } \operatorname{or} \operatorname{sign}(\psi(s))=-1 \mu \text {-a.e. }
$$

From equation (A.7) it follows that $\mu$-a.e.

$$
|\psi(s)| \leq \rho^{-1} \int K(t, s)|\psi(t)| d \mu(t)
$$

Multiplying both sides by $\varphi(s)$, integrating and applying (A.6) yields

$$
\int|\psi(s)| \varphi(s) d \mu(s) \leq \rho^{-1} \iint K(t, s) \varphi(s)|\psi(t)| d \mu(t) d \mu(s)=\int|\psi(t)| \varphi(t) d \mu(t) .
$$

It follows that $\mu$-a.e.

$$
|\psi(s)|=\rho^{-1} \int K(t, s)|\psi(t)| d \mu(t),
$$

i.e. $|\psi|$ is an eigenfunction of $L^{*}$. 
Next, equation $|\psi(s)|=\psi(s) \operatorname{sign}(\psi(s))$ implies that $\mu$-a.e.

$$
\rho^{-1} \int K(t, s)|\psi(t)| d \mu(t)=\rho^{-1} \int K(t, s) \psi(t) d \mu(t) \operatorname{sign}(\psi(s)) .
$$

It follows that for a.e. $(t, s)$ under $\mu \times \mu$

$$
|\psi(t)|=\psi(t) \operatorname{sign}(\psi(s))
$$

By the positivity condition on $K,|\psi|>0 \mu$-a.e. by the same reasoning as given in step (4). Thus, A.8 follows. Q.E.D.

\section{D.5 Proof of Theorem 13.}

Note that $K(c, s)=r(c, s) s^{-\gamma_{0}} f(s, c) /[f(s) f(c)]>0$ almost everywhere by $r(c, s)>0$ and $f(s, c)>0$ almost everywhere. Therefore the conclusion follows from Lemma A.4 with $f(s) d s=$ $d \mu(s)$. Q.E.D.

\section{E Tangential Cone Conditions}

In this Appendix we discuss some inequalities that are related to identification of $\alpha_{0}$. Throughout this Appendix we maintain that $m\left(\alpha_{0}\right)=0$. Define

$$
\begin{aligned}
\mathcal{N} & =\{\alpha: m(\alpha) \neq 0\}, \quad \mathcal{N}^{\prime}=\left\{\alpha: m^{\prime}\left(\alpha-\alpha_{0}\right) \neq 0\right\}, \\
\mathcal{N}_{\eta}^{\prime} & =\left\{\alpha:\left\|m(\alpha)-m^{\prime}\left(\alpha-\alpha_{0}\right)\right\|_{\mathcal{B}} \leq \eta\left\|m^{\prime}\left(\alpha-\alpha_{0}\right)\right\|_{\mathcal{B}}\right\}, \eta>0, \\
\mathcal{N}_{\eta} & =\left\{\alpha:\left\|m(\alpha)-m^{\prime}\left(\alpha-\alpha_{0}\right)\right\|_{\mathcal{B}} \leq \eta\|m(\alpha)\|_{\mathcal{B}}\right\}, \quad \eta>0 .
\end{aligned}
$$

Here $\mathcal{N}$ can be interpreted as the identified set and $\mathcal{N}^{\prime}$ as the set where the rank condition holds. The set $\mathcal{N}_{\eta}^{\prime}$ is a set on which an inequality version of equation (2.2) holds. The inequality used to define $\mathcal{N}_{\eta}$ is similar to the tangential cone condition from the literature on computation in nonlinear ill-posed inverse problems, e.g. Hanke, Neubauer and Scherzer (1995) and Dunker et. al. (2012).

The following results gives some relations among these sets: 
Lemma A.5: For any $\eta>0$,

$$
\mathcal{N}_{\eta} \cap \mathcal{N}^{\prime} \subset \mathcal{N}, \quad \mathcal{N}_{\eta}^{\prime} \cap \mathcal{N} \subset \mathcal{N}^{\prime}
$$

If $0<\eta<1$ then

$$
\mathcal{N}_{\eta} \cap \mathcal{N} \subset \mathcal{N}^{\prime}, \quad \mathcal{N}_{\eta}^{\prime} \cap \mathcal{N}^{\prime} \subset \mathcal{N}
$$

Proof: Note that $\alpha \in \mathcal{N}_{\eta}$ and the triangle inequality gives

$$
-\|m(\alpha)\|_{\mathcal{B}}+\left\|m^{\prime}\left(\alpha-\alpha_{0}\right)\right\|_{\mathcal{B}} \leq \eta\|m(a)\|_{\mathcal{B}}
$$

so that $\|m(\alpha)\|_{\mathcal{B}} \geq(1+\eta)^{-1}\left\|m^{\prime}\left(\alpha-\alpha_{0}\right)\right\|_{\mathcal{B}}$. Therefore if $\alpha \in \mathcal{N}_{\eta} \cap \mathcal{N}^{\prime}$ we have $\|m(\alpha)\|_{\mathcal{B}}>0$, i.e. $\alpha \in \mathcal{N}$, giving the first conclusion. Also, if $\alpha \in \mathcal{N}_{\eta}^{\prime}$ we have

$$
-\left\|m^{\prime}\left(\alpha-\alpha_{0}\right)\right\|_{\mathcal{B}}+\|m(\alpha)\|_{\mathcal{B}} \leq \eta\left\|m^{\prime}\left(\alpha-\alpha_{0}\right)\right\|_{\mathcal{B}}
$$

so that $\left\|m^{\prime}\left(\alpha-\alpha_{0}\right)\right\|_{\mathcal{B}} \geq(1+\eta)^{-1}\|m(\alpha)\|_{\mathcal{B}}$. Therefore, if $\alpha \in \mathcal{N}_{\eta}^{\prime} \cap \mathcal{N}$ we have $\left\|m^{\prime}\left(\alpha-\alpha_{0}\right)\right\|_{\mathcal{B}}>$ 0 , giving the second conclusion.

Next, for $0<\eta<1$ and $\alpha \in \mathcal{N}_{\eta}$ we have

$$
\|m(\alpha)\|_{\mathcal{B}}-\left\|m^{\prime}\left(\alpha-\alpha_{0}\right)\right\|_{\mathcal{B}} \leq \eta\|m(\alpha)\|_{\mathcal{B}}
$$

so that $\left\|m^{\prime}\left(\alpha-\alpha_{0}\right)\right\| \geq(1-\eta)\|m(\alpha)\|_{\mathcal{B}}$. Therefore, if $\alpha \in \mathcal{N}_{\eta} \cap \mathcal{N}$ we have $\left\|m^{\prime}\left(\alpha-\alpha_{0}\right)\right\|_{\mathcal{B}}>0$, giving the third conclusion. Similarly, for $0<\eta<1$ and $\alpha \in \mathcal{N}_{\eta}^{\prime}$ we have

$$
\left\|m^{\prime}\left(\alpha-\alpha_{0}\right)\right\|_{\mathcal{B}}-\|m(\alpha)\|_{\mathcal{B}} \leq \eta\left\|m^{\prime}\left(\alpha-\alpha_{0}\right)\right\|_{\mathcal{B}}
$$

so that $\|m(\alpha)\|_{\mathcal{B}} \geq(1-\eta)\left\|m^{\prime}\left(\alpha-\alpha_{0}\right)\right\|_{\mathcal{B}}$. Therefore if $\alpha \in \mathcal{N}_{\eta}^{\prime} \cap \mathcal{N}^{\prime}$ we have $\|m(\alpha)\|_{\mathcal{B}}>0$, giving the fourth conclusion. Q.E.D.

The first conclusion shows that when the tangential cone condition is satisfied the set on which the rank condition holds is a subset of the identified set. The second condition is less interesting, but does show that the rank condition is necessary for identification when $\alpha \in \mathcal{N}_{\eta}^{\prime}$. The third conclusion shows that the rank condition is also necessary for identification under the tangential cone condition for $0<\eta<1$. The last conclusion shows that when $\alpha \in \mathcal{N}_{\eta}^{\prime}$ with $0<\eta<1$ the rank condition is sufficient for identification. 
When the side condition that $\alpha \in \mathcal{N}_{\eta}$ or $\alpha \in \mathcal{N}_{\eta}^{\prime}$ are imposed for $0<\eta<1$, the rank condition is necessary and sufficient for identification.

Corollary A.6: If $0<\eta<1$ then

$$
\mathcal{N}_{\eta} \cap \mathcal{N}^{\prime}=\mathcal{N}_{\eta} \cap \mathcal{N}, \quad \mathcal{N}_{\eta}^{\prime} \cap \mathcal{N}^{\prime}=\mathcal{N}_{\eta}^{\prime} \cap \mathcal{N}
$$

Proof: By intersecting both sides of the first conclusion of Lemma A.5 with $\mathcal{N}_{\eta}$ we find that $\mathcal{N}_{\eta} \cap \mathcal{N}^{\prime} \subset \mathcal{N}_{\eta} \cap \mathcal{N}$. For $\eta<1$ it follows similarly from the third conclusion of Lemma A.5 that $\mathcal{N}_{\eta} \cap \mathcal{N} \subset \mathcal{N}_{\eta} \cap \mathcal{N}^{\prime}$, implying $\mathcal{N}_{\eta} \cap \mathcal{N}^{\prime}=\mathcal{N}_{\eta} \cap \mathcal{N}$, the first conclusion. The second conclusion follows similarly. Q.E.D.

The equalities in the conclusion of this result show that the rank condition (i.e. $\alpha \in \mathcal{N}^{\prime}$ ) is necessary and sufficient for identification (i.e. $\alpha \in \mathcal{N}$ ) under either of the side conditions that

$$
\alpha \in \mathcal{N}_{\eta}^{\prime} \text { or } \alpha \in \mathcal{N}_{\eta}, 0<\eta<1
$$

In parametric models Rothenberg (1971) showed that when the Jacobian has constant rank in a neighborhood of the true parameter the rank condition is necessary and sufficient for local identification. These conditions fill an analogous role here, in the sense that when $\alpha$ is restricted to either set, the rank condition is necessary and sufficient for identification.

The sets $\mathcal{N}_{\eta}$ and $\mathcal{N}_{\eta}^{\prime}$ are related to each other in the way shown in the following result.

LEMmA A.7. If $0<\eta<1$ then $\mathcal{N}_{\eta} \subset \mathcal{N}_{\eta /(1-\eta)}^{\prime}$ and $\mathcal{N}_{\eta}^{\prime} \subset \mathcal{N}_{\eta /(1-\eta)}$.

Proof: By the triangle inequality

$$
\begin{aligned}
\left\|m^{\prime}\left(\alpha-\alpha_{0}\right)\right\|_{\mathcal{B}} & \leq\left\|m(\alpha)-m^{\prime}\left(\alpha-\alpha_{0}\right)\right\|_{\mathcal{B}}+\|m(\alpha)\|_{\mathcal{B}}, \\
\|m(\alpha)\|_{\mathcal{B}} & \leq\left\|m(\alpha)-m^{\prime}\left(\alpha-\alpha_{0}\right)\right\|_{\mathcal{B}}+\left\|m^{\prime}\left(\alpha-\alpha_{0}\right)\right\|_{\mathcal{B}} .
\end{aligned}
$$

Therefore, for $\alpha \in \mathcal{N}_{\eta}$,

$$
\left\|m(\alpha)-m^{\prime}\left(\alpha-\alpha_{0}\right)\right\|_{\mathcal{B}} \leq \eta\left\|m(\alpha)-m^{\prime}\left(\alpha-\alpha_{0}\right)\right\|_{\mathcal{B}}+\eta\left\|m^{\prime}\left(\alpha-\alpha_{0}\right)\right\|_{\mathcal{B}}
$$

Subtracting $\eta\left\|m(\alpha)-m^{\prime}\left(\alpha-\alpha_{0}\right)\right\|_{\mathcal{B}}$ from both sides and dividing by $1-\eta$ gives $\alpha \in \mathcal{N}_{\eta /(1-\eta)}^{\prime}$. The second conclusion follows similarly. Q.E.D. 


\section{References}

[1] Ai, C. and X. Chen (2003) "Efficient Estimation of Models with Conditional Moment Restrictions Containing Unknown Functions," Econometrica 71, 1795-1844.

[2] Anderson, R. and W.R. Zame (2000), "Genericity with Infinitely Many Parameters," Advances in Theoretical Economics 1, 1-62.

[3] Andrews, D.W.K. (2011) "Examples of L2-Complete and Boundedly-Complete Distributions," Cowles Foundation Discussion Paper No. 1801, Yale University.

[4] Blundell, R., X. Chen, and D. Kristensen (2007), "Semi-Nonparametric IV Estimation of ShapeInvariant Engel Curves," Econometrica 75 1613-1669.

[5] Carrasco, M., J.P. Florens, and E. Renault (2007), "Linear Inverse Problems in Structural Econometrics: Estimation Based on Spectral Decomposition and Regularization," In: Heckman,J.J. and E.E. Leamer, eds., Handbook of Econometrics, vol. 6B, North Holland.

[6] Chamberlain, G. (1986) "Asymptotic Efficiency in Semiparametric Models with Censoring," Journal of Econometrics 32, 1986, 189-218.

[7] Chamberlain, G. (1987) "Asymptotic Efficiency in Estimation with Conditional Moment Restrictions," Journal of Econometrics 34, 305-334.

[8] Chamberlain, G. (1992) "Efficiency Bounds for Semiparametric Regression," Econometrica 60, 567 $-596$.

[9] Chen, X. and S. Ludvigson (2009) "Land of Addicts? An Empirical Investigation of Habit-Based Asset Pricing Models," Journal of Applied Econometrics 24, 1057-1093.

[10] Chen, X. and D. Pouzo (2009) "Efficient Estimation of Semiparametric Conditional Moment Models with Possibly Nonsmooth Residuals," Journal of Econometrics 152, 46-60.

[11] Chen, X., and D. Pouzo (2012) "Estimation of Nonparametric Conditional Moment Models with Possibly Nonsmooth Generalized Residuals," Econometrica 80, 277-321.

[12] Chen, X. and M. Reiß (2011) "On Rate Optimality for Ill-Posed Inverse Problems in Econometrics," Econometric Theory, 27, 497-521.

[13] Chernozhukov, V., and C. Hansen (2005) "An IV Model of Quantile Treatment Effects. Econometrica $73,245-261$.

[14] Chernozhukov, V., G.W. Imbens, and W.K. Newey (2007) "Instrumental Variable Estimation of Nonseparable Models," Journal of Econometrics 139, 4-14.

[15] Cochrane, J. (2005) Asset Pricing, Princeton University Press.

[16] Dudley, R. (1989) Real analysis and probability. Pacific Grove, California: Wadsworth \& Brooks/Cole. 
[17] Darolles, S, Y. Fan, J.-P. Florens, and E. Renault (2011) "Nonparametric Instrumental Regression," Econometrica, 79, 1541-1565.

[18] Dunker, F., J.P. Florens, T. Hohage, J. Johannes, E. Mammen (2012) "Iterative Estimation of Solutions to Noisy Nonlinear Operator Equations in Nonparametric Instrumental Regression," working paper.

[19] Engl, H. W., M. Hanke, and A. Neubauer (1996) Regularization of Inverse Problems. Dordrecht: Kluwer.

[20] Escanciano, J.C. and S. Hoderlein (2010) "Nonparametric Identification of Euler Equations," working paper.

[21] Fisher, F. (1966) The Identification Problem in Econometrics,New York: McGraw-Hill Book Co.

[22] Florens, J.-P., J. Johannes, and S. Van Bellegem (2011) "Identification and Estimation by Penalization in Nonparametric Instrumental Regression," Econometric Theory, 27, 472-496.

[23] Florens, J.-P., J. Johannes, and S. Van Bellegem (2012) "Instrumental Regression in Partially Linear Models," The Econometrics Journal 15, 304-324.

[24] Florens, J.-P., M. Mouchart, and J.M. Rolin (1990) Elements of Bayesian Statistics, Dekker, New York.

[25] Florens, J.-P., and E. Sbai (2010) "Local Identification in Empirical Games of Incomplete Information," Econometric Theory 26, $1638-1662$.

[26] Gallant, A.R. and G. Tauchen (1989) "Semiparametric Estimation of Conditional Constrained Heterogenous Processes: Asset Pricing Applications," Econometrica 57, 1091-1120.

[27] Hall, P. and J. Horowitz (2005): "Nonparametric Methods for Inference in the Presence of Instrumental Variables," Annals of Statistics 33, 2904-2929.

[28] Hall, R.E. (1978) "Stochastic Implications of the Life Cycle-Permanent Income Hypothesis: Theory and Evidence," Journal of Political Economy 86, 971-987.

[29] Hanke, M., A. Neubauer, and O. Scherzer (1995) "A Convergence Analysis of Landweber Iteration for Nonlinear Ill-Posed Problems." Numer. Math., 72.??

[30] Hansen, L.P. (1982) "Large Sample Properties of Generalized Method of Moments Estimators," Econometrica 50, 1029-1054.

[31] Hansen, L.P. (2012): "Dynamic Valuation Decomposition within Stochastic Economies," Econometrica 80, 911-967. Fisher-Schultz Lecture at the European Meetings of the Econometric Society.

[32] Hansen, L.P. and J. Scheinkman (2009): "Long-Term Risk: An Operator Approach," Econometrica $77,117-234$. 
[33] Hansen, L.P. and J. Scheinkman (2012): "Recursive Utility in a Markov Environment with Stochastic Growth," Proceedings of the National Academy of Sciences 109, 11967-11972.

[34] Hansen, L.P. and K.J. Singleton. (1982): "Generalized Instrumental Variables Estimation of Nonlinear Rational Expectations Models," Econometrica 50, 1269-1286.

[35] Hansen L.P., J. Heaton, J. Lee, N. Roussanov (2007) "Intertemporal Substitution and Risk Aversion," In: Heckman,J.J. and E.E. Leamer, eds., Handbook of Econometrics, Elsevier, Volume 6, 3967-4056.

[36] D'Haultfoeuille, X. (2011) "On the Completeness Condition in Nonparametric instrumental regression," Econometric Theory 27, 460-471.

[37] Horowitz, J. L., and S. Lee (2007) "Nonparametric Instrumental Variables Estimation of a Quantile Regression Model," Econometrica, 75, 1191-1208.

[38] Krein, M. G. and Rutman, M. A. (1950) Linear operators leaving invariant a cone in a Banach space. Amer. Math. Soc. Translation 1950, no. 26.

[39] Kress, R. (1999) Linear Integral Equations, Second Edition, New York: Springer-Verlag.

[40] Ledoux, M. and Talagrand, M. (2011) Probability in Banach spaces. Isoperimetry and processes. Springer-Verlag, Berlin.

[41] Lewbel, A., O. Linton and S. Srisuma (2012) "Nonparametric Euler Equation Identification and Estimation," working paper.

[42] Luenberger, D. (1969) Optimization by Vector Space Methods, New York: John Wiley \& Sons.

[43] Marcus, M.B and Pisier, G. (1981) Random Fourier Series with Applications to Harmonic Analysis. Princeton University Press.

[44] Mattner, L., (1993) "Some Incomplete But Boundedly Complete Location Families,". Annals of Statistics 21, 2158-2162.

[45] Newey, W.K. and J.L. Powell (1988) "Instrumental Variables Estimation for Nonparametric Models," Working Paper Version of Published Paper, MIT.

[46] Newey, W.K. and J.L. Powell (2003) "Instrumental Variables Estimation for Nonparametric Models," Econometrica 71, 1565-1578.

[47] Rothenberg, T.J., (1971) "Identification in Parametric Models," Econometrica 39, 577-591.

[48] Santos, A. (2011) "Instrumental Variables Methods for Recovering Continuous Linear Functionals," Journal of Econometrics 161, 129-146.

[49] Sargan, D. (1983), "Identification and Lack of Identification," Econometrica 51, 1605-1634.

[50] Zimmer, R. (1990) Essential Results of Functional Analysis, Chicago Lectures in Mathematics, Chicago. 